\title{
Hydrogen atom transfer methodology for the synthesis of C-22, C-23, and C-25 stereoisomers of cephalostatin north 1 side chain from spirostan sapogenins
}

\author{
Carmen Betancor, ${ }^{b}$ Raimundo Freire, ${ }^{a}$ Ine's Pe' rez-Mart'in, ${ }^{\text {a Thierry Prange }}{ }^{\prime c}$ and Ernesto Sua' rez $^{\mathrm{a}, *}$ \\ anstituto de Productos Naturales y Agrobiolog'za del C.S.I.C., Carretera de La Esperanza 3, 38206 La Laguna, Tenerife, Spain

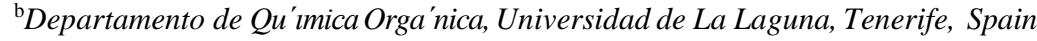 \\ ${ }^{\mathrm{c}}$ LURE, Universite' Paris-Sud, Paris, 91405 Orsay Cedex, France
}

Received 12 November 2004; revised 18 January 2005; accepted 20 January 2005

\begin{abstract}
A simple synthesis of all eight C-22, C-23, and C-25 diastereoisomers of the cephalostatin north 1 side chain has been accomplished from (25R)-5a-spirostan-3b-ol (tigogenin). The synthesis involves selective hydroxylations at C-23 and C-25 and reductive opening of the 1,6-dioxaspiro[4.5]decane spirostan system to give a conveniently protected 5a-furostan-3b,23,25,26-tetrol. The construction of the required 1,6-dioxaspiro[4.4]nonane system entailed an intramolecular hydrogen abstraction reaction promoted by the C-25 alkoxyl radical as the key step. Acid-catalyzed isomerization of the spiroketal unit suggested that $22 R$ isomers are the thermodynamic products while the $22 S$ isomers are the result of kinetic control. The acid-catalyzed equilibrium between 1,6-dioxaspiro[4.4]nonane and 1,6dioxaspiro[4.5]decane systems was also studied. In the 1,6-dioxaspiro[4.4]nonane units, the observed ${ }^{3} J_{23,24}$ coupling constants suggest that the five-membered puckered ring-F undergoes substantial conformational changes on going from $22 S$ to $22 R$ isomers.
\end{abstract}

1.

\section{Introduction}

Cephalostatins ${ }^{1}$ and the structurally related ritterazines ${ }^{2}$ comprise a group of secondary metabolites isolated from marine invertebrates (Cephalodiscus gilchristi and Ritterella tokioka, respectively) which have attracted considerable attention from synthetic organic chemists and pharmacologists due to their complex structures and significant biological properties. .3 They are alkaloids constituted by two steroidal units linked through a pyrazine ring involving the $\mathrm{C} 2-\mathrm{C} 3$ position of each monomeric unit and are among the most potent cytotoxins ever isolated from a natural source. In most of these substances the steroidal eightcarbon side chain has been transformed into a 1,6-dioxaspiro [4.4]nonane system. In particular, a polyoxygenated $(2 S, 4 R, 5 S, 9 S)$-2-hydroxymethyl-2,9-dimethyl-1,6-dioxaspiro [4.4]nonan-4-ol substructure is found in the side chain of the north unit in many cephalostatins (17 out of 19), and the majority of ritterazines have a 2,2,9-trimethyl-1,6-dioxaspiro[4.4]nonane system on one or other side of their skeletons (Fig. 1).

Keywords: Cephalostatin; Radical reaction; Hydrogen abstraction; Alkoxyl radical; Steroid; Spirostan sapogenin.

* Corresponding author. Tel.: C 34922 251004; fax: C 34922 260135;

e-mail: esuarez@ipna.csic.es
The syntheses of several of these natural products and analogues have been achieved ${ }^{4}$ and during these studies very interesting methodologies have been brought to light. $\underline{5}$ Nevertheless, despite efforts by several research groups, the mechanism of biological action remains unknown. - The structure-activity relationship between cephalostatins and OSW-1 (Fig. 1), a related cholestane glycoside isolated from a terrestrial plant (Ornithogalum saundersiae), ${ }^{7}$ supports the hypothesis that the bioactive intermediate might be an oxocarbenium ion located at rings $\mathrm{E}$ or $\mathrm{F}$ and originated by opening the dioxaspiro grouping. $\cdot \frac{6,7 b, 8}{\mathrm{We}} \mathrm{Can}$ deduce from this that the stereochemistries at C-22, C-23, and $\mathrm{C}-25$, which doubtless have a strong influence on the conformation and stability of the dioxaspiro[4.4]nonane system, may also influence the activity of cephalostatins.

With these ideas in mind, we decided to develop a simple methodology to permit the synthesis of all eight possible isomers of this system by modification of the steroidal side chain of a commercially available spirostan sapogenin, ${ }^{2}$ the key step being the formation of the spiroketal system by an intramolecular hydrogen abstraction reaction (IHA) promoted by alkoxyl radicals. $\frac{10}{}$ In previous papers from this laboratory we have demonstrated the utility of IHA reactions in the synthesis of dioxaspiro[4.4]nonane ring systems in the carbohydrate field. ${ }^{11}$ From this previous 

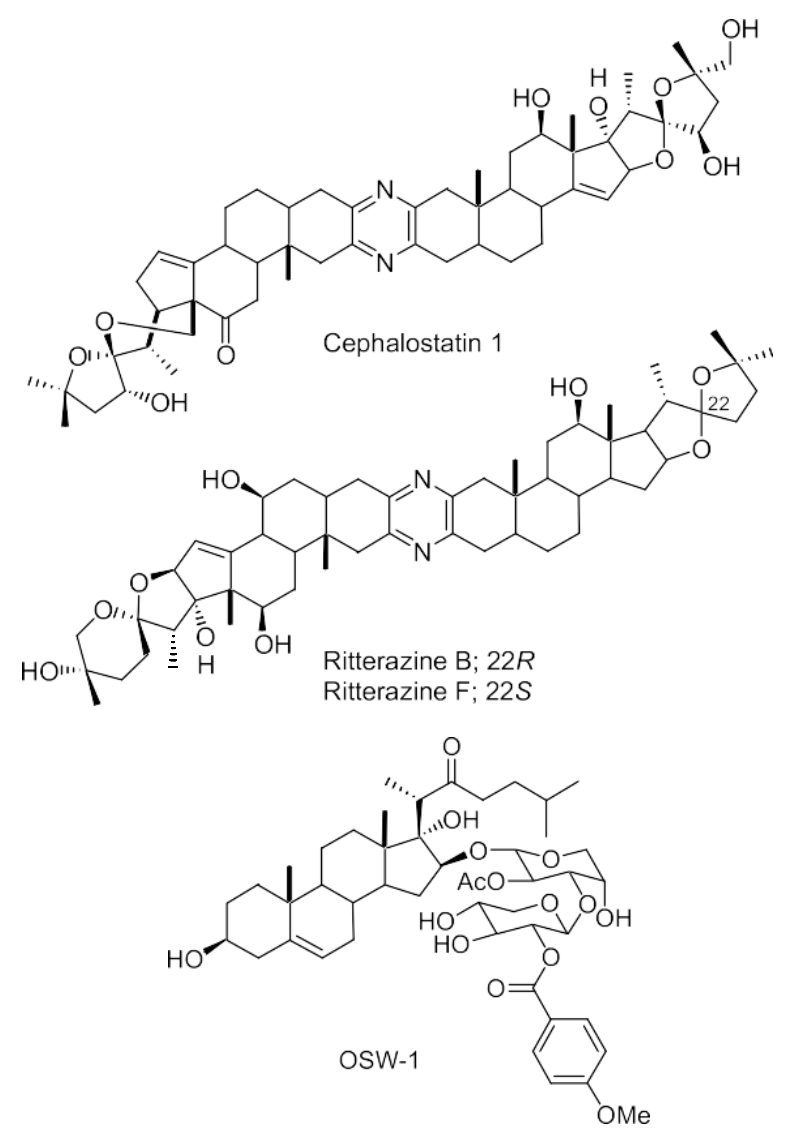

Figure 1. Examples of representative cephalostatins and reitterazines.

experience we were confident that both spiroketal isomers could be obtained using this methodology. This is synthetically important because in most of the ritterazines both stereoisomers at the spiroketal center were obtained from the natural source. $\frac{12}{}$

\section{Results and discussion}

The synthesis began with 3-methoxy-23-oxotigogenin (2) (Scheme 1) prepared by using a previously described procedure via oxidation of 3-methoxy-tigogenin (1) with $\mathrm{NaNO}_{2} / \mathrm{BF}_{3} \$ E t_{2} \mathrm{O} . \underline{13}$ The reduction of 2 with L-selectride furnished a mixture of alcohols 3 and 4 (72\%, 1.7:1) from which the alcohol 3 with the correct natural orientation $(23 R)$ could be obtained in moderate yield. The reduction of 2 with $\mathrm{NaBH}_{4}$ afforded preferentially the alcohol $4(23 S)$ with the non-natural stereochemistry $(91 \%, 19: 1)$.

The two C-23 diastereoisomers 3 and 4 were taken through the following steps of the synthesis separately (Scheme 1). The tigogenin dioxaespiro[4.5]decane system present in 3 was regio- and stereoselectively reduced with $\mathrm{Ph}_{2} \mathrm{SiH}_{2} /$ $\mathrm{TiCl}_{4}$ to give the diol $5-R . \stackrel{14}{-}$ Conversion of $5-R$ to the monoprotected secondary alcohol $8-R$ was accomplished by a three-step protection-deprotection sequence involving formation of the primary pivalate $6-R$, silylation of the 23alcohol with TBDMSOTf, and hydrolysis of pivalate $7-R$ with $\mathrm{KOH}$ in methanol. Nitrophenylselenenylation of the primary alcohol in $8-R$ followed by oxidative elimination furnished alkene $10-R . \underline{15}$ In a series of reactions identical to
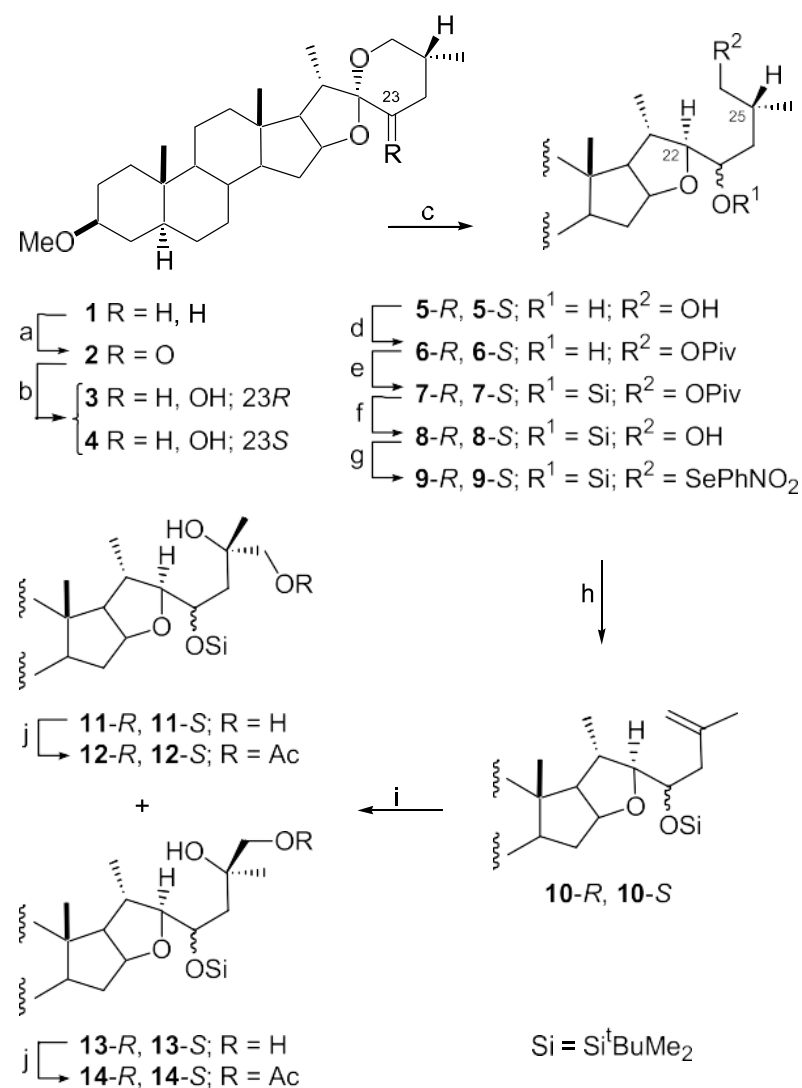

10-R, 10-S

$\mathrm{Si}=\mathrm{Si}^{\mathrm{t}} \mathrm{BuMe}_{2}$

Scheme 1. Reagents and conditions: (a) $\mathrm{NaNO}_{2}, \mathrm{BF}_{3} \$ \mathrm{Et}_{2} \mathrm{O}, \mathrm{AcOH}, \mathrm{rt}, 1$ h, 68\%; (b) $\mathrm{NaBH}_{4}, \mathrm{EtOH}, \mathrm{rt}, 1 \mathrm{~h}, 91 \%$ (3/4 ratio 5:95) or L-selectride, THF, K20 8C, 1.5 h, $72 \%$ (3/4 ratio 63:37); (c) $\mathrm{Ph}_{2} \mathrm{SiH}_{2}, \mathrm{TiCl}_{4}, \mathrm{CH}_{2} \mathrm{Cl}_{2}$, $\mathrm{K} 20$ 8C;

(d) pivaloyl chloride, $\mathrm{Py}, \mathrm{CH}_{2} \mathrm{Cl}_{2}, \mathrm{rt}, 6-R 95 \%, 6-\mathrm{S} 97 \%$; (e) ${ }^{\mathrm{t}} \mathrm{BuMe}_{2} \mathrm{SiOTf}$, $\mathrm{CH}_{2} \mathrm{Cl}_{2}, \mathrm{Et}_{3} \mathrm{~N}$, rt, 7-R 81\%, 7-S 98\%; (f) $\mathrm{KOH}, \mathrm{MeOH}, \mathrm{rt}, 8-R 92 \%, 8-S$ 91\%; (g) $o-\mathrm{NO}_{2} \mathrm{PhSeCN}, n-\mathrm{Bu}_{3} \mathrm{P}$, THF, rt, 9-R 99\%, 9-S 97\%; (h) $\mathrm{H}_{2} \mathrm{O}_{2}$, THF, rt, 10-R 92\%, 10-S 82\%; (i) $\mathrm{OsO}_{4}, \mathrm{Py}, \mathrm{CH}_{2} \mathrm{Cl}_{2}$, rt; (j) $\mathrm{Ac}_{2} \mathrm{O}$, Py, rt. [For yields of the (i) and (j) reactions, see supplementary data section]. The $(R, S)$ designs the stereochemistry at $\mathrm{C}-23$.

those described (Scheme 1), the $23 S$ isomer 4 was converted into $10-S$ via $5-S, 6-S, 7-S, 8-S$, and $9-S$. Stoichiomeric osmylation of the 10- $R$ olefin afforded an inseparable mixture of diols 11-R and 13- $R$ which could be separated after acetylation of the primary alcohol 12- $R$ and 14- $R$ in a $1: 2$ ratio $(99 \%)$. In contrast, the osmylation of the $10-S$ isomer afforded a separable mixture of diols 11-S and 13-S in a 2:1 ratio $(98 \%)$, which were subsequently and separately acetylated to give $12-S$ and $14-S$.

Initials attempts to asymmetrically dihydroxylate the 25olefin were unsuccessful. $\frac{16}{1}$ Using the Corey $(1 S, 2 S)$ $N^{1}, N^{2}$-bis(mesitylmethyl)-1,2-diphenyl-1,2-ethanediamine reagent, $\frac{17}{-}$ the $10-R$ olefin gave the diols with similar yield and diastereomeric ratio $(11-R / 13-R, 1: 2,97 \%)$ compared with the uncatalyzed reaction. As both isomeric diols were required for this study the uncatalyzed osmylation reaction was preferred.

The IHA reaction was carried out by separately treating compounds 12-R, 12-S, 14- $R$, and 14-S with (diacetoxyiodo) benzene and iodine under irradiation with two $80 \mathrm{~W}$ tungsten-filament lamps at 50 8C (Scheme 2). The alcohols that possess the natural stereochemistry at C$23(R)$ 12- $R$ and 14- $R$ gave 1,6-dioxaspiro[4.4]nonane compounds 15 


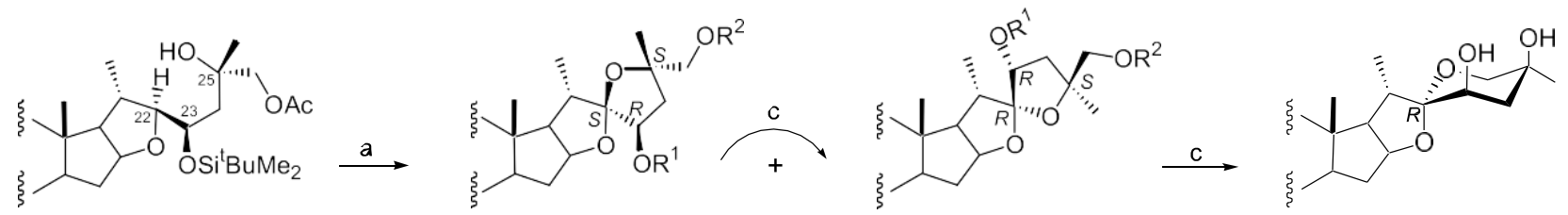

$12 R$

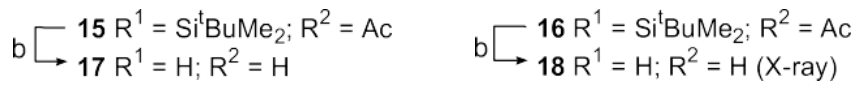

19
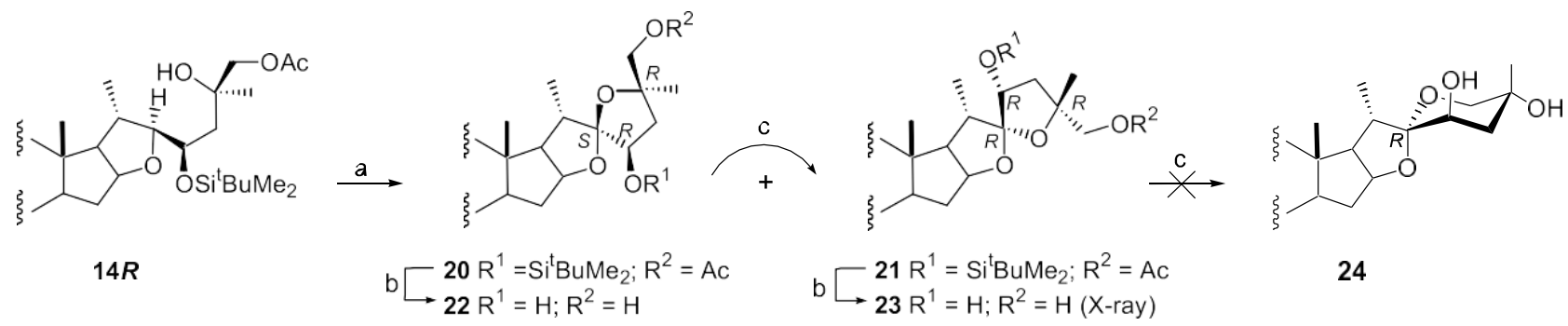

24
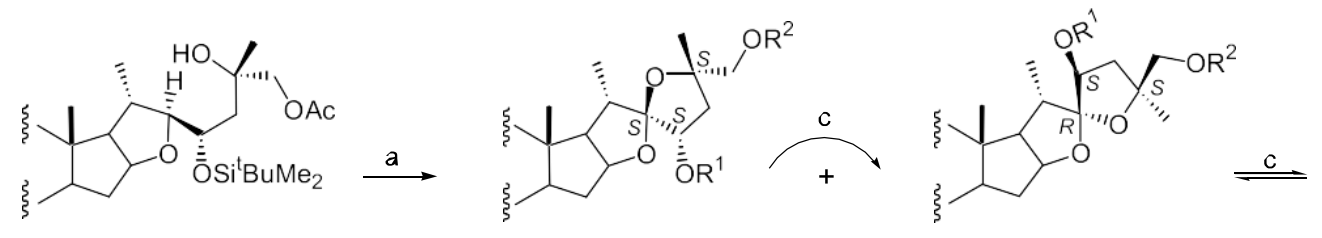

$12 S$

$$
\begin{array}{rlrl}
\mathrm{b} L 25 \mathrm{R}^{1}=\mathrm{Si}^{\mathrm{t} B u M e} & ; \mathrm{R}^{2}=\mathrm{Ac} & \mathrm{b} L 26 \mathrm{R}^{1}=\mathrm{Si}^{\mathrm{t} B u M \mathrm{~B}_{2}} ; \mathrm{R}^{2}=\mathrm{Ac} \\
\mathrm{2} 27 \mathrm{R}^{1}=\mathrm{H} ; \mathrm{R}^{2}=\mathrm{H} & & 28 \mathrm{R}^{1}=\mathrm{H} ; \mathrm{R}^{2}=\mathrm{H}
\end{array}
$$

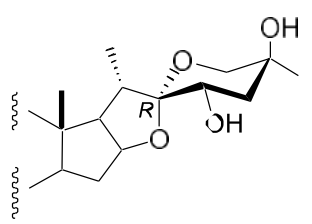

29 (X-ray)
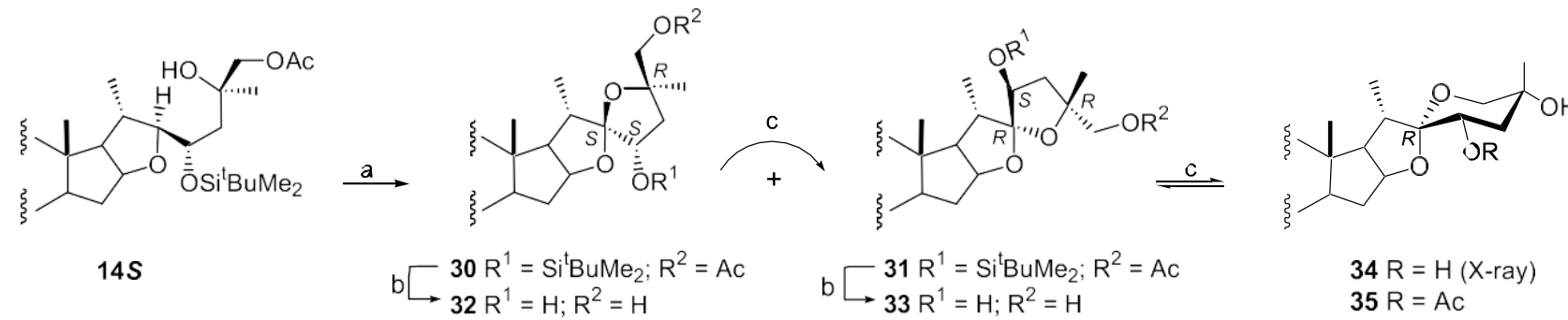

Scheme 2. Reagents and conditions: (a) $\mathrm{PhI}(\mathrm{OAc})_{2}, \mathrm{I}_{2}$, cyclohexane, $h \mathrm{n}, 70$ 8C, 15 23\%, 16 60\%; 20 28\%, 21 55\%; 25 23\%, 26 74\%; 30 28\%, 31 47\%; (b) (i)

TBAF, THF, rt, (ii) $\mathrm{KOH}, \mathrm{MeOH}$, rt (yields too steps: 17 62\%, 18 73\%, 22 74\%, 23 70\%, 27 67\%, 28 87\%, 32 99\%, 33 91\%); (c) $\mathrm{H}^{\mathrm{C}}$, $\mathrm{CH}_{2} \mathrm{Cl}_{2}$, rt.

and $16(83 \%, 28: 72)$ and 20 and $21(83 \%, 33: 67)$, respectively. On the other hand, the alcohols with the inverted stereochemistry at C-23 $(S)$ 12-S and 14-S afforded after the IHA reaction dioxaspiro compounds 25, and 26 $(97 \%$,

24:76) and 30 and 31 (75\%, 37:63), respectively. The protecting groups of the alcohols at C-23 and C-25 were selected to favor the 1,5-hydrogen atom transfer reaction. A weak electron-withdrawing group (EWG) at C-23 (silyl ether) should favor the hydrogen abstraction and the subsequent oxidation of the $\mathrm{C}-22$ radical to the oxocarbenium ion intermediate. $\frac{18}{}$ Also the stronger EWG at C-26 (acetyl ester) should prevent the competitive b-fragmentation of the alkoxyl radical. The choice of the protecting group could be critical and a hypothetical model where the two protecting groups have been interchanged (acetyl at C-23 and silyl at C-26) should give significant amounts of the methyl ketone from $b$-fragmentation. $\frac{19}{}$ The desired diols $17,18,22,23,27,28,32$, and 33 were obtained by hydrolysis of the silyl and acetyl protective groups. The structures of these eight stereoisomers of the cephalostatine north 1 side chain were determined by extensive ${ }^{1} \mathrm{H}$ and ${ }^{13} \mathrm{C}$ NMR 1D and 2D studies including DEPT, COSY, HMBC, and HSQC experiments. Using 2D NOESY and DNOE, the relative stereochemistry of the newly created stereogenic centers (C-22 and C-25) with respect to the known stereochemistry of the alcohol at C-23 may be assigned in each case..$^{20}$ As the flexibility of the 1,6-dioxaspiro system (vide infra) may introduce some uncertainty in the NOE results, the structure and stereochemistry were subsequently confirmed by X-ray crystallographic analysis of compounds 18 and 23. ${ }^{21}$ The $(22 S, 23 R, 25 S)$-diol 17 possesses the stereochemistry of the natural cephalostatins. Compounds $17,22,27$, and 32 appear to be the products of kinetic control whereas 18, 23, 28, and 33 are the thermodynamic products. The relative stability of these compounds was determined by following the evolution of the acid-catalyzed rearrangement through a C-22 oxocarbenium ion. Due to the presence of the 25,26-glycol, dioxaspiro compounds of the 1,6-dioxaspiro[4.5]decane type (e.g. 19) may also be formed. $\underline{22}$

In a preliminary experiment, diol 17 was transformed into the $22 R$-isomer 18 and both 17 and 18 finally led to the dioxaspiro[4.5]decane 19 under prolonged acid treatment 
(Scheme 2). Subsequently, it was established that, in the 1,6-dioxaspiro[4.4]nonane system the $22 S$ isomers 17,22 , 27 , and 32 are easily transformed, under mild acid conditions, into the $22 R$ isomers $18,23,28$, and 33 , respectively, confirming that the $22 R$ are the most stable compounds.

The transformation from the dioxaspiro [4.4]nonane to the [4.5]decane system deserves further comment. $\frac{23}{3}$ Although compound 19 is obtained in moderate yield by acidcatalyzed isomerization of diol 18, we observed that even under prolonged reaction times neither 22 nor 23 yielded the corresponding dioxaspiro[4.5]-compound 24 to any appreciable extent. Furthermore, the reactions of 28 and 33 under similar conditions reach an equilibrium (28/29, $60: 40$ and $33 / 35,66: 34)$ after several hours at room temperature.

Aware that iodine is a Lewis acid, we also explored the iodine-catalyzed isomerization of $22 R$-isomers and similar results to those obtained with protic acids were achieved. For example, reaction of diol 27 in cyclohexane with iodine $(10 \mathrm{mM})$ under the same conditions of the IHA reaction afforded after $1 \mathrm{~h}$ at $708 \mathrm{C}$ the equilibrium mixture of 28 and 29 in a ratio of 60:40. Analogously, 32 was isomerized to a mixture of 33 and 34 (60:40) under the same conditions. These findings suggest that a possible iodine-catalyzed partial isomerization between $22 S$ and $22 R$ isomers may well have occurred during the IHA reaction. Partial isomerization at the spirocenter may indeed be accom- plished by treatment of fully protected compound 25 with iodine under conditions emulating the IHA reaction, to give a mixture of 25 and 26 (2:8) after 4 $h$ at $708 \mathrm{C}$.

These finding are in agreement with the results of a MM2 study (Table 1), compounds 17, 24, 27, and 32 being the highest energy isomers in the respective series while 19 , 23, 28, and 33 are the most stable. $\frac{24}{}$ The isomeric pairs 28 and 29, and 33 and 34 have similar energy (DEZ0.4$0.6 \mathrm{Kcal} /$

Table 1. Structural characteristics of dioxaspiro compounds

\begin{tabular}{|c|c|c|c|c|}
\hline \multirow[t]{2}{*}{ Compound } & \multirow[t]{2}{*}{$\mathrm{D} E^{\mathrm{a}}(\mathrm{kcal} / \mathrm{mol})$} & \multicolumn{2}{|c|}{$P^{\mathrm{b}}(\text { conformation })^{\mathrm{c}}$} & \multirow[t]{2}{*}{${ }^{3} J_{23,24} \mathrm{~d}(\mathrm{~Hz})$} \\
\hline & & E-ring & F-ring & \\
\hline 17 & 6.8 & $134\left(E_{16}\right)$ & $146\left({ }^{23} T_{22}\right)$ & $8.1,8.4$ \\
\hline 17 (X-ray) $^{\mathrm{e}}$ & - & $127\left(E_{16}\right)$ & $145\left({ }^{23} T_{22}\right)$ & - \\
\hline 18 & 3.4 & $102\left({ }^{\circ} T_{16}\right)$ & $339\left(E_{23}\right)$ & $0.0,5.7$ \\
\hline 18 (X-ray) & - & $93\left({ }^{\circ} E\right)$ & $332\left({ }^{22} T_{23}\right)$ & - \\
\hline 19 & 0.0 & $96\left({ }^{\circ} E\right)$ & $-\left({ }^{22} C_{25}\right)$ & $2.8,2.8$ \\
\hline 22 & 2.9 & $141\left({ }^{17} T_{16}\right)$ & $155\left({ }^{23} E\right)$ & $8.1,9.8$ \\
\hline 23 & 0.0 & $85\left({ }^{\circ} E\right)$ & $320\left({ }^{22} T_{23}\right)$ & $0.0,5.6$ \\
\hline 23 (X-ray) & - & $90\left({ }^{\circ} E\right)$ & $324\left({ }^{22} T_{23}\right)$ & - \\
\hline 24 & 4.3 & $99\left({ }^{\circ} E\right)$ & $-\left({ }^{22} C_{25}\right)$ & - \\
\hline 27 & 4.4 & $148\left({ }^{17} T_{16}\right)$ & $144\left({ }^{23} T_{22}\right)$ & $0.0,4.9$ \\
\hline 28 & 0.0 & $89\left({ }^{\circ} E\right)$ & $346\left(E_{23}\right)$ & $8.3,10.5$ \\
\hline 29 & 0.4 & $89\left({ }^{\circ} E\right)$ & $-\left({ }^{22} C_{25}\right)$ & $5.3,11.6$ \\
\hline 29 (X-ray) & - & $80\left({ }^{\circ} T_{22}\right)$ & $-\left({ }^{22} C_{25}\right)$ & - \\
\hline 32 & 4.6 & $145\left({ }^{17} T_{16}\right)$ & $153\left({ }^{23} T_{22}\right)$ & $0.0,4.6$ \\
\hline 33 & 0.0 & $85\left({ }^{\circ} E\right)$ & $320\left({ }^{22} T_{23}\right)$ & $8.5,9.8$ \\
\hline 34 & 0.6 & $91\left({ }^{\circ} E\right)$ & $-\left({ }^{22} C_{25}\right)$ & $5.3,11.7$ \\
\hline 34 (X-ray) & - & $72\left({ }^{\circ} T_{22}\right)$ & $-\left({ }^{22} C_{25}\right)$ & - \\
\hline
\end{tabular}

\footnotetext{
${ }^{\mathrm{a}}$ Changes of the relative MM2 energy (in $\mathrm{kcal} / \mathrm{mol}$ ) with respect to the lowest energy isomer in the respective series.

b Altone-Sundaralingam phase angle (in degrees) as defined in Ref. $29 \mathrm{c}$.

c An adaptation of the IUPAC nomenclature of carbohydrates is used (Ref. $\underline{30}$ ).

${ }^{\mathrm{d}}$ Experimental ${ }^{3} J_{\mathrm{HH}}$ coupling from $500 \mathrm{MHz}$ spectra.

e Data were taken from the X-ray analysis of $(22 S, 23 R, 25 S)$-3b,12b-diacetoxy-22,25-epoxy-5a-furostan-23,26-diol (Ref. 27).
}

(ca. 60:40) is reached after extended periods of time. compounds are shown in Table 1. For instance, compounds with the same stereochemistry at C-23 display significantly different coupling constants between the protons $\mathrm{H}_{23}$ and $\mathrm{H}_{24}$ on changing from the $22 R$ to the $22 S$ series of compounds (compare the coupling constant of 17 with 18 or 22 with 23 in which the stereochemistry at C-23 is always $R$, or 27 with 28 and 32 with 33 where the stereochemistry is $23 S)$. The small couplings $(0,5 \mathrm{~Hz})$ suggest a pseudoaxial orientation of the $\mathrm{C}-23$ alcohol (e.g. 18) while the larger couplings $(8,10 \mathrm{~Hz})$ are more consistent with a pseudo- equatorially disposed alcohol (e.g. 17)..$\underline{25}$ Nevertheless, a reasonable explanation for this phenomenon is necessarily associated with a change of the conformation of the tetrahydrofuran F-ring on going from the $22 S$ to $22 R$ series of compounds (Fig. 2). The study of the conformation of this 1,6-dioxaspiro ring system may not be an easy task due to the significant flexibility of the puckered five-membered rings, although, in this case, some conformational con- straint, exerted by the substituents and the fused D-ring, may be expected. ${ }^{26}$ In this approach we have determined the conformations of the E- and F-rings of the eight different

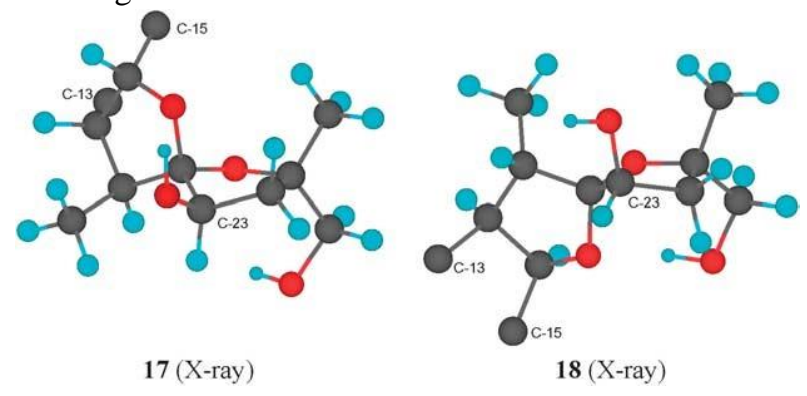

Figure 2. Ring F conformations of 17 and 18, taken from the X-ray crystal structures. For the sake of clarity only E and F rings are shown.
Several other interesting features in the structure of these 
isomers over minimized structures (MM2) using the X-ray structures $17, \underline{27} 18$, and 23 (X-ray) as starting geometry. $\underline{28}$ With this study we are not attempting to make a complete conformational analysis of the 1,6-dioxaspiro system, but simply to explain the apparently anomalous coupling constants observed for the proton at C-23.

The structures of lowest energy calculated by this methodology have E- and F-ring conformations that were very similar to those found in the crystallographic structures. A comparison of the conformations from the crystal structure with those established by molecular mechanics calculations is presented in Table 1 [compare 17 with 17 (X-ray), 18 with 18 (X-ray), and 23 with 23 (X-ray)]. The ring conformations have been described by the AltoneSundaralingam phase angle $\frac{29}{}$ and the IUPAC conformational nomenclature for the furanose form of monosaccharides has been adapted to these rings. $\underline{30}$ The E-ring of the $22 S$-isomers $(17,22,27$, and 32$)$ adopts a preferred conformation $E_{16}$ or ${ }^{17} T_{16}(P Z 134-1488)$ in the southern hemisphere of the pseudorotational itinerary of the ring (Table 1). ${ }^{29}$ The E-ring conformation in the $22 R$ series of isomers $(18,23,28$, and

33) is located in the east ${ }^{\circ} E(P Z 102-858)$ of the pseudorotational wheel. On the other hand, the F-ring of the $22 S$-isomers adopts a preferred conformation ${ }^{23} T_{22}$ or ${ }^{23} E$ (PZ144-1558) in the southern hemisphere, while, conformations ${ }^{22} T^{23}$ or $E^{23}(P Z 320-3468)$ in the northern hemisphere are found for the F-ring of the $22 R$-isomers.

The experimental ${ }^{3} J_{23,24} \mathrm{H}-\mathrm{H}$ coupling constants were measured in $500 \mathrm{MHz}$ spectra (Table 1), and are in agreement with those calculated over minimized structures using the HLA equation, $\frac{31}{}$ the largest individual discrepancy between experimental and calculated constants being $1.4 \mathrm{~Hz}$.

In the 1,6-dioxa[4.5]decane compounds 19, 29 and 34, firm evidence in favor of a ${ }^{22} C_{25}$ conformation for the Fring was obtained by the ${ }^{3} J_{23,24}$ coupling constants. The alternative ${ }^{25} C_{22}$ chair conformation can be ruled out on the basis of the same measurements (Table 1). $\underline{32}$ X-ray diffraction analysis confirmed the ${ }^{22} C_{25}$ conformation in the solid state for 29 and $34 . \underline{21}$

\section{Conclusion}

In summary, we have demonstrated the usefulness of the IHA reaction in the construction of the steroidal 1,6dioxaspiro[4.4]nonane ring system. $\underline{33}$ Since thermodynamically less stable isomers at the spirocenter can be obtained, this methodology should be especially useful in the synthesis of the natural products when both isomers are isolated from nature, as described for several ritterazines. $\underline{12}$

The preparation of all eight possible isomers has led to the discovery that the spirocenter stereochemistry can profoundly influence the conformation of the F-ring. Taking such an effect into account, the apparently anomalous coupling constant for the proton at $\mathrm{C}-23$, observed in the NMR spectra of these compounds, can be readily explained. From these findings the question that now arises is whether the conformation of the F-ring might influence the biological activity, as occurs in other types of tetrahydrofuran derivatives. ${ }^{29 c, 34}$ In any case, this should be taken into consideration in the development of new biologically active cephalostatin and ritterazin analogs.

Although we are aware that our conclusions regarding the stability and conformation of the spiroketal side chain in the different series of these simple monomers may not be fully extrapolatable to the bioactive products, we believe that they could help in designing such compounds.

\section{Experimental}

\subsection{General methods}

Melting points were determined with a hot-stage apparatus and are uncorrected. Optical rotations were measured at the sodium line at ambient temperature in $\mathrm{CHCl}_{3}$ solutions. IR spectra were recorded in $\mathrm{CHCl}$ solutions unless otherwise stated. NMR spectra were determined at $500 \mathrm{MHz}$ for ${ }^{1} \mathrm{H}$ and $125.7 \mathrm{MHz}$ for ${ }^{13} \mathrm{C}$ in $\mathrm{CDCl}_{3}$ unless otherwise stated, in the presence of TMS as internal standard. Mass spectra were determined at $70 \mathrm{eV}$. Merck silica gel 60 PF (0.063$0.2 \mathrm{~mm}$ ) was used for column chromatography. Circular layers of $1 \mathrm{~mm}$ of Merck silica gel $60 \mathrm{PF}_{254}$ were used on a Chromatotron for centrifugally assisted chromatography. Commercially available reagents and solvents were analytical grade or were purified by standard procedures prior to use. All reactions involving air- or moisture-sensitive materials were carried out under a nitrogen atmosphere. The spray reagents for TLC analysis were conducted with $0.5 \%$ vanillin in $\mathrm{H} \mathrm{SO}-\mathrm{EtOH}(4: 1)$ and further heating until development of color.

4.1.1. $(22 S, 23 R, 25 S)$-3b-Methoxy-23-tertbutyldimethyl- silyloxy-26-acetoxy-22,25-epoxy-5afurostan (15) and $(22 R, 23 R, 25 S)-3 \mathrm{~b}$-methoxy-23tert-butyldimethylsilyl- oxy-26-acetoxy-22,25epoxy-5a-furostan (16). A solution of the alcohol 12- $R$ $(60 \mathrm{mg}, 0.096 \mathrm{mmol})$ in cyclohexane $(10 \mathrm{~mL})$ containing (diacetoxyiodo)benzene (40 mg,

$0.124 \mathrm{mmol})$ and iodine $(25 \mathrm{mg}, 0.098 \mathrm{mmol})$ was irradiated with two $80 \mathrm{~W}$ tungsten-filament lamps at $508 \mathrm{C}$ for

$3.5 \mathrm{~h}$. The reaction mixture was then poured into aqueous solution of sodium thiosulfate $(10 \%)$ and extracted with $\mathrm{Et}_{2} \mathrm{O}$. The combined organic extracts were washed with brine, dried $\left(\mathrm{Na}_{2} \mathrm{SO}_{4}\right)$ and concentrated. Chromatotron chromatography (hexanes-EtOAc, 95:5) of the residue afforded compound compound 15 (14 mg, 0.022 mmol,

23.4\%) and 16 (36 mg, $0.058 \mathrm{mmol}, 60 \%$ ). Compound 15: amorphous; [a] $]_{\mathrm{D}} \mathrm{K} 18$ (c 0.23$)$; IR $1746 \mathrm{~cm}^{\mathrm{K} 1}$; ${ }^{1} \mathrm{H}$ NMR $0.07(3 \mathrm{H}, \mathrm{s}), 0.09(3 \mathrm{H}, \mathrm{s}), 0.64(1 \mathrm{H}, \mathrm{m}), 0.81(3 \mathrm{H}, \mathrm{s}), 0.87$ $(3 \mathrm{H}, \mathrm{s}), 0.90(9 \mathrm{H}, \mathrm{s}), 1.06(3 \mathrm{H}, \mathrm{d}, J \mathrm{Z} 7.2 \mathrm{~Hz}), 1.32(3 \mathrm{H}, \mathrm{s})$, $1.91(1 \mathrm{H}, \mathrm{dd}, J Z 11.0,11.4 \mathrm{~Hz}), 2.03(1 \mathrm{H}, \mathrm{dd}, J Z 7.6$, $11.6 \mathrm{~Hz}), 2.08(3 \mathrm{H}, \mathrm{s}), 2.30(1 \mathrm{H}$, dddd, JZ7.0, 7.0, 7.0, $7.0 \mathrm{~Hz}), 3.12(1 \mathrm{H}$, dddd, JZ4.6, 4.6, 10.9, $10.9 \mathrm{~Hz}), 3.35$ (3H, s), 3.88 (2H, s), $4.30(1 \mathrm{H}, \mathrm{dd}, J Z 7.8,10.4 \mathrm{~Hz}), 4.62$ (1H, ddd, JZ7.1, 7.1, 7.1 Hz); ${ }^{13} \mathrm{C}$ NMR K5.4 $\left(\mathrm{CH}_{3}\right), \mathrm{K} 4.0$ $\left(\mathrm{CH}_{3}\right), 12.3\left(\mathrm{CH}_{3}\right), 16.1\left(\mathrm{CH}_{3}\right), 16.6\left(\mathrm{CH}_{3}\right), 17.8(\mathrm{C}), 20.9$ $\left(\mathrm{CH}_{2}\right), 21.1\left(\mathrm{CH}_{3}\right), 25.8\left(4 ! \mathrm{CH}_{3}\right), 27.9\left(\mathrm{CH}_{2}\right), 28.8\left(\mathrm{CH}_{2}\right)$, $32.3\left(\mathrm{CH}_{2}\right), 32.4\left(\mathrm{CH}_{2}\right), 34.3\left(\mathrm{CH}_{2}\right), 35.0(\mathrm{CH}), 35.9(\mathrm{C})$, $36.9\left(\mathrm{CH}_{2}\right), 37.5(\mathrm{CH}), 40.2\left(\mathrm{CH}_{2}\right), 40.3\left(\mathrm{CH}_{2}\right), 41.1(\mathrm{C})$, $44.8(\mathrm{CH}), 54.5(\mathrm{CH}), 55.5\left(\mathrm{CH}_{3}\right), 55.6(\mathrm{CH}), 61.6(\mathrm{CH})$, 
$70.3\left(\mathrm{CH}_{2}\right), 73.2(\mathrm{CH}), 79.0(\mathrm{C}), 79.8(\mathrm{CH}), 81.7(\mathrm{CH})$, $117.9(\mathrm{C}), 170.6(\mathrm{C}) ; \mathrm{MS} \mathrm{m} / \mathrm{z}$ (rel intensity) $618\left(\mathrm{M}^{\mathrm{C}}, ! 1\right)$, 561 (6), 475 (30), 287 (23); HRMS calcd for $\mathrm{C}_{36} \mathrm{H}_{62} \mathrm{O}_{6} \mathrm{Si}$ 618.4316; found 618.4255. Anal. Calcd for $\mathrm{C}_{36} \mathrm{H}_{62} \mathrm{O}_{6} \mathrm{Si}$ : C, 69.86; H, 10.10. Found: C, 69.01; H, 10.17. Compound 16: amorphous; [a] $]_{\mathrm{D}} \mathrm{K} 45$ (c 0.24); IR $1745 \mathrm{~cm}^{\mathrm{K} 1} ;{ }^{1} \mathrm{H}$ NMR $0.07(3 \mathrm{H}, \mathrm{s}), 0.08(3 \mathrm{H}, \mathrm{s}), 0.62(1 \mathrm{H}, \mathrm{m}), 0.76(3 \mathrm{H}, \mathrm{s}), 0.80$ $(3 \mathrm{H}, \mathrm{s}), 0.90(9 \mathrm{H}, \mathrm{s}), 1.06(3 \mathrm{H}, \mathrm{d}, J \mathrm{Z} 6.9 \mathrm{~Hz}), 1.33(3 \mathrm{H}, \mathrm{s})$, $1.59(1 \mathrm{H}, \mathrm{dd}, J Z 0.0,13.3 \mathrm{~Hz}), 1.93(1 \mathrm{H}$, ddd, $J Z 5.7,7.5$, $12.4 \mathrm{~Hz}), 2.05(3 \mathrm{H}, \mathrm{s}), 2.22(1 \mathrm{H}, \mathrm{dd}, J Z 5.4,13.3 \mathrm{~Hz}), 2.32$ (1H, dddd, $J Z 6.1,6.1,6.1,6.1 \mathrm{~Hz}), 3.11(1 \mathrm{H}$, dddd, $J Z 4.5$, $4.5,10.8,10.8 \mathrm{~Hz}), 3.33(3 \mathrm{H}, \mathrm{s}), 3.88(1 \mathrm{H}, \mathrm{d}, J Z 10.9 \mathrm{~Hz})$, $4.10(1 \mathrm{H}, \mathrm{d}, J Z 10.9 \mathrm{~Hz}), 4.14(1 \mathrm{H}, \mathrm{d}, J Z 4.6 \mathrm{~Hz}), 4.44(1 \mathrm{H}$, ddd, $J Z 5.6,7.8,7.8 \mathrm{~Hz}) ;{ }^{13} \mathrm{C}$ NMR K5.1 $\left(\mathrm{CH}_{3}\right), \mathrm{K} 5.0$ $\left(\mathrm{CH}_{3}\right), 12.3\left(\mathrm{CH}_{3}\right), 16.3\left(\mathrm{CH}_{3}\right), 16.8\left(\mathrm{CH}_{3}\right), 17.9(\mathrm{C}), 20.9$ $\left(\mathrm{CH}_{2}\right), 21.0\left(\mathrm{CH}_{3}\right), 25.0\left(\mathrm{CH}_{3}\right), 25.7\left(3 ! \mathrm{CH}_{3}\right), 27.9\left(\mathrm{CH}_{2}\right)$, $28.7\left(\mathrm{CH}_{2}\right), 32.0\left(\mathrm{CH}_{2}\right), 32.2\left(\mathrm{CH}_{2}\right), 34.3\left(\mathrm{CH}_{2}\right), 35.3(\mathrm{CH})$, $35.9(\mathrm{C}), 36.2(\mathrm{CH}), 36.9\left(\mathrm{CH}_{2}\right), 39.8\left(\mathrm{CH}_{2}\right), 41.0(\mathrm{C}), 42.3$ $\left(\mathrm{CH}_{2}\right), 44.7(\mathrm{CH}), 54.4(\mathrm{CH}), 55.5\left(\mathrm{CH}_{3}\right), 56.3(\mathrm{CH}), 63.1$ $(\mathrm{CH}), 70.8\left(\mathrm{CH}_{2}\right), 78.5(\mathrm{CH}), 79.8(\mathrm{CH}), 81.3(\mathrm{CH}), 82.0$ (C), 120.9 (C), $171.0(\mathrm{C}) ; \mathrm{MS} \mathrm{m} / \mathrm{z}$ (rel intensity) $618\left(\mathrm{M}^{\mathrm{C}}\right.$, ! 1 ), 561 (2), 545 (7), 475 (32), 287 (43); HRMS calcd for $\mathrm{C}_{36} \mathrm{H}_{62} \mathrm{O}_{6} \mathrm{Si}$ 618.4316; found 618.4238. Anal. Calcd for $\mathrm{C}_{36} \mathrm{H}_{62} \mathrm{O}_{6} \mathrm{Si}$ : C, 69.86; H, 10.10. Found: C, 69.93; H, 10.22.

\subsection{2. (22S,23R,25S)-3b-Methoxy-22,25-epoxy-5a-} furo- stan-23,26-diol (17). To a solution of compound 15 (13 mg, $0.021 \mathrm{mmol})$ in THF ( $3 \mathrm{~mL})$ was added TBAF $(0.1 \mathrm{~mL}, 0.1 \mathrm{mmol}, 1.0 \mathrm{M}$ in THF) and stirred at room temperature for $2 \mathrm{~h}$. The mixture was then poured into aqueous saturated solution of $\mathrm{NaHCO}_{3}$ and extracted with AcOEt. The organic extracts were washed with brine, dried $\left(\mathrm{Na}_{2} \mathrm{SO}_{4}\right)$ and concentrated. Chromatotron chromatography (benzene-EtOAc, 90:10) of the residue afforded $(22 S, 23 R, 25 S)$-3b-methoxy-26-acetoxy-22,25-epoxy-5afurostan-23-ol (8.6 mg, $0.017 \mathrm{mmol}, 81 \%)$ : $\mathrm{mp} \mathrm{151-154} \mathrm{8C}$ (from EtOAc); IR 3447, $1744 \mathrm{~cm}^{\mathrm{K} 1} ;{ }^{1} \mathrm{H}$ NMR $0.64(1 \mathrm{H}, \mathrm{m})$, $0.81(3 \mathrm{H}, \mathrm{s}), 0.91(3 \mathrm{H}, \mathrm{s}), 1.13(3 \mathrm{H}, \mathrm{d}, J Z 7.5 \mathrm{~Hz}), 1.29(3 \mathrm{H}$, s), $2.01(1 \mathrm{H}, \mathrm{ddd}, J Z 5.7,7.2,12.3 \mathrm{~Hz}), 2.07(3 \mathrm{H}, \mathrm{s}), 2.27$ $(1 \mathrm{H}, \mathrm{dd}, J Z 7.8,12.6 \mathrm{~Hz}), 2.34(1 \mathrm{H}$, dddd, $J Z 3.5,7.4,7.4$, $7.4 \mathrm{~Hz}), 3.11(1 \mathrm{H}$, dddd, $J Z 4.6,4.6,10.9,10.9 \mathrm{~Hz}), 3.33$ $(3 \mathrm{H}, \mathrm{s}), 3.86(2 \mathrm{H}, \mathrm{s}), 4.25(1 \mathrm{H}, \mathrm{ddd}, J Z 8.8,8.8,8.8 \mathrm{~Hz})$, $4.54(1 \mathrm{H}$, ddd, $J Z 7.0,7.0,7.0 \mathrm{~Hz}) ;{ }^{13} \mathrm{C}$ NMR $12.3\left(\mathrm{CH}_{3}\right)$, $16.1\left(\mathrm{CH}_{3}\right), 17.5\left(\mathrm{CH}_{3}\right), 20.9\left(\mathrm{CH}_{3}\right), 20.9\left(\mathrm{CH}_{2}\right), 25.8\left(\mathrm{CH}_{3}\right)$, $27.9\left(\mathrm{CH}_{2}\right), 28.8\left(\mathrm{CH}_{2}\right), 32.4\left(\mathrm{CH}_{2}\right), 34.0\left(\mathrm{CH}_{2}\right), 34.3$ $\left(\mathrm{CH}_{2}\right), 34.8(\mathrm{CH}), 35.9(\mathrm{C}), 36.9\left(\mathrm{CH}_{2}\right), 39.1(\mathrm{CH}), 40.1$ $\left(\mathrm{CH}_{2}\right), 41.5(\mathrm{C}), 41.9\left(\mathrm{CH}_{2}\right), 44.8(\mathrm{CH}), 54.5(\mathrm{CH}), 55.48$ $(\mathrm{CH}), 55.52\left(\mathrm{CH}_{3}\right), 63.3(\mathrm{CH}), 70.2\left(\mathrm{CH}_{2}\right), 73.0(\mathrm{CH}), 78.9$ (C), $79.8(\mathrm{CH}), 83.7(\mathrm{CH}), 118.5(\mathrm{C}), 170.7(\mathrm{C}) ; \mathrm{MS} \mathrm{m} / \mathrm{z}$ (rel intensity) $486\left(\mathrm{M}^{\mathrm{C}} \mathrm{KH}_{2} \mathrm{O}, 3\right), 471$ (! 1 ), $426(4), 413$ (4), 361 (39), 287 (100); HRMS calcd for $\mathrm{C}_{30} \mathrm{H}_{46} \mathrm{O}_{5}$ 486.3345; found 486.3363. Anal. Calcd for $\mathrm{C}_{30} \mathrm{H}_{48} \mathrm{O}_{6}: \mathrm{C}$, 71.39; H, 9.59. Found: C, 71.51; H, 9.71. A solution of this acetate $(8 \mathrm{mg}, 0.0158 \mathrm{mmol})$ in $\mathrm{MeOH}(5 \mathrm{~mL})$ containing $\mathrm{KOH}(0.15 \mathrm{~g})$ was stirred at room temperature for $4 \mathrm{~h}$. The mixture was poured into water and extracted with AcOEt. The combined extracts were washed with brine, dried $\left(\mathrm{Na}_{2} \mathrm{SO}_{4}\right)$ and concentrated. Chromatotron chromatography (hexanes-EtOAc, 7:3) of the residue afforded compound 17 (5.6 mg, $0.012 \mathrm{mmol}, 76 \%$ ): $\mathrm{mp} \mathrm{187.5-190} \mathrm{8C} \mathrm{(from}$ EtOAc- $n$-hexane); [a] $]_{\mathrm{D}} \mathrm{C} 11$ ( c 0.19); IR $3417 \mathrm{~cm}^{\mathrm{K} 1}$; ${ }^{1} \mathrm{H}$ NMR $0.64(1 \mathrm{H}, \mathrm{m}), 0.81(3 \mathrm{H}, \mathrm{s}), 0.92(3 \mathrm{H}, \mathrm{s}), 1.13(3 \mathrm{H}, \mathrm{d}$, $J Z 7.4 \mathrm{~Hz}), 1.27(3 \mathrm{H}, \mathrm{s}), 1.69(1 \mathrm{H}, \mathrm{dd}, J Z 8.4,12.6 \mathrm{~Hz})$,
$2.02(1 \mathrm{H}$, ddd, $J Z 5.6,7.3,12.4 \mathrm{~Hz}), 2.20(1 \mathrm{H}$, br d, $J Z$ $10.0 \mathrm{~Hz}), 2.29(1 \mathrm{H}, \mathrm{dd}, J Z 8.1,12.6 \mathrm{~Hz}), 2.34(1 \mathrm{H}$, dddd, $J Z 4.0,7.5,7.5,7.5 \mathrm{~Hz}), 3.12$ (1H, dddd, $J Z 4.7,4.7,11.0$, $11.0 \mathrm{~Hz}), 3.29(1 \mathrm{H}, \mathrm{d}, J Z 11.3 \mathrm{~Hz}), 3.34(3 \mathrm{H}, \mathrm{s}), 3.38(1 \mathrm{H}$, d, $J Z 11.3 \mathrm{~Hz}), 4.22(1 \mathrm{H}$, ddd, $J Z 8.4,8.4,8.4 \mathrm{~Hz}), 4.56$ (1H, ddd, JZ7.0, 7.0, 8.7 Hz); ${ }^{13} \mathrm{C}$ NMR $12.3\left(\mathrm{CH}_{3}\right), 16.2$ $\left(\mathrm{CH}_{3}\right), 17.5\left(\mathrm{CH}_{3}\right), 20.9\left(\mathrm{CH}_{2}\right), 25.3\left(\mathrm{CH}_{3}\right), 27.8\left(\mathrm{CH}_{2}\right), 28.7$ $\left(\mathrm{CH}_{2}\right), 32.3\left(\mathrm{CH}_{2}\right), 33.9\left(\mathrm{CH}_{2}\right), 34.3\left(\mathrm{CH}_{2}\right), 34.8(\mathrm{CH}), 35.9$ (C), $36.9\left(\mathrm{CH}_{2}\right), 39.4(\mathrm{CH}), 40.1\left(\mathrm{CH}_{2}\right), 41.5(\mathrm{C}), 41.7$ $\left(\mathrm{CH}_{2}\right), 44.8(\mathrm{CH}), 54.5(\mathrm{CH}), 55.4\left(\mathrm{CH}_{3}\right), 55.5(\mathrm{CH}), 63.4$ $(\mathrm{CH}), 69.7\left(\mathrm{CH}_{2}\right), 73.3(\mathrm{CH}), 79.8(\mathrm{CH}), 81.3(\mathrm{C}), 83.7$ (CH), $118.6(\mathrm{C}) ; \mathrm{MS} \mathrm{m} / z$ (rel intensity) $461\left(\mathrm{M}^{\mathrm{C}} \mathrm{KH}, ! 1\right.$ ), 431 (4), 287 (100); HRMS calcd for $\mathrm{C}_{28} \mathrm{H}_{45} \mathrm{O}_{5}$ 461.3267; found 461.3225. Anal. Calcd for $\mathrm{C}_{28} \mathrm{H}_{46} \mathrm{O}_{5}: \mathrm{C}, 72.69 ; \mathrm{H}$, 10.02. Found: $\mathrm{C}, 72.81 ; \mathrm{H}, 10.19$.

\subsection{3. $(22 R, 23 R, 25 S)$-3b-Methoxy-22,25-epoxy-5a-} furo- stan-23,26-diol (18). To a solution of compound 16 (35 mg, $0.056 \mathrm{mmol})$ in THF $(5 \mathrm{~mL})$ was added TBAF $(0.3 \mathrm{~mL}, 0.3 \mathrm{mmol}, 1.0 \mathrm{M}$ in THF) and stirred at room temperature for $3 \mathrm{~h}$. The mixture was then poured into aqueous saturated solution of $\mathrm{NaHCO}_{3}$ and extracted with $\mathrm{Et}_{2} \mathrm{O}$. The organic extracts were washed with brine, dried $\left(\mathrm{Na}_{2} \mathrm{SO}_{4}\right)$ and concentrated. Chromatotron chromatography (hexanes-EtOAc, 80:20) of the residue afforded $(22 R, 23 R, 25 S)$-3b-methoxy-26-acetoxy-22,25-epoxy-5afurostan-23-ol (23 mg, $0.045 \mathrm{mmol}, 81 \%)$ : $\mathrm{mp} 208.5-209$ 8C (from EtOAc- $n$-hexane); [a] $]_{\mathrm{D}} \mathrm{K} 57$ (c 1.03); IR 3516, $1723 \mathrm{~cm}^{\mathrm{K} 1} ;{ }^{1} \mathrm{H}$ NMR $0.62(1 \mathrm{H}, \mathrm{m}), 0.77(3 \mathrm{H}, \mathrm{s}), 0.80(3 \mathrm{H}$, s), $1.09(3 \mathrm{H}, \mathrm{d}, J Z 6.9 \mathrm{~Hz}), 1.34(3 \mathrm{H}, \mathrm{s}), 1.93(1 \mathrm{H}, \mathrm{ddd}$, $J Z 5.8,7.4,12.6 \mathrm{~Hz}), 2.07(3 \mathrm{H}, \mathrm{s}), 2.28(1 \mathrm{H}, \mathrm{dd}, J Z 5.6$, $13.7 \mathrm{~Hz}), 2.39(1 \mathrm{H}$, dddd, JZ6.4, 6.4, 6.4, $6.4 \mathrm{~Hz}), 3.11$ (1H, dddd, $J Z 4.6,4.6,10.9,10.9 \mathrm{~Hz}), 3.33(3 \mathrm{H}, \mathrm{s}), 3.93$ $(1 \mathrm{H}, \mathrm{d}, J Z 10.9 \mathrm{~Hz}), 4.09(1 \mathrm{H}, \mathrm{d}, J Z 10.9 \mathrm{~Hz}), 4.18(1 \mathrm{H}, \mathrm{br}$ d, $J Z 4.9 \mathrm{~Hz}), 4.44(1 \mathrm{H}$, ddd, $J Z 5.7,7.8,7.8 \mathrm{~Hz}) ;{ }^{13} \mathrm{C}$ NMR $12.3\left(\mathrm{CH}_{3}\right), 16.1\left(\mathrm{CH}_{3}\right), 16.8\left(\mathrm{CH}_{3}\right), 20.9\left(\mathrm{CH}_{3}\right), 21.0$ $\left(\mathrm{CH}_{2}\right), 25.5\left(\mathrm{CH}_{3}\right), 27.9\left(\mathrm{CH}_{2}\right), 28.7\left(\mathrm{CH}_{2}\right), 31.9\left(\mathrm{CH}_{2}\right), 32.2$ $\left(\mathrm{CH}_{2}\right), 34.3\left(\mathrm{CH}_{2}\right), 35.2(\mathrm{CH}), 35.9(\mathrm{C}), 36.1(\mathrm{CH}), 36.9$ $\left(\mathrm{CH}_{2}\right), 39.7\left(\mathrm{CH}_{2}\right), 41.0(\mathrm{C}), 42.0\left(\mathrm{CH}_{2}\right), 44.8(\mathrm{CH}), 54.4$ $(\mathrm{CH}), 55.5\left(\mathrm{CH}_{3}\right), 56.3(\mathrm{CH}), 63.3(\mathrm{CH}), 70.9\left(\mathrm{CH}_{2}\right), 78.1$ $(\mathrm{CH}), 79.8(\mathrm{CH}), 81.2(\mathrm{CH}), 81.6(\mathrm{C}), 120.2(\mathrm{C}), 171.0(\mathrm{C})$; MS $m / z$ (rel intensity) $504\left(\mathrm{M}^{\mathrm{C}} ! 1\right.$ ), 486 (11), 431 (6), 287 (100); HRMS calcd for $\mathrm{C}_{30} \mathrm{H}_{48} \mathrm{O}_{6}$ 504.3451; found 504.3455. Anal. Calcd for $\mathrm{C}_{30} \mathrm{H}_{48} \mathrm{O}_{6}: \mathrm{C}, 71.39 ; \mathrm{H}, 9.59$. Found: C, 71.06; H, 9.86. A solution of this acetate $(20 \mathrm{mg}$, $0.0397 \mathrm{mmol})$ in $\mathrm{MeOH}(10 \mathrm{~mL})$ containing $\mathrm{KOH}(0.35 \mathrm{~g})$ was stirred at room temperature for $4 \mathrm{~h}$. The mixture was poured into water and extracted with AcOEt. The combined extracts were washed with brine, dried $\left(\mathrm{Na}_{2} \mathrm{SO}_{4}\right)$ and concentrated. Chromatotron chromatography (hexanesEtOAc, 7:3) of the residue afforded compound 18 (16.5 $\mathrm{mg}, 0.036 \mathrm{mmol}, 90 \%$ ): $\mathrm{mp} 211.5-213.58 \mathrm{C}$ (from EtOAc$n$-hexane); [a] $]_{\mathrm{D}} \mathrm{K} 55$ (c 0.108); IR 3426, $1453 \mathrm{~cm}^{\mathrm{K} 1} ;{ }^{1} \mathrm{H}$ NMR $0.63(1 \mathrm{H}, \mathrm{m}), 0.78(3 \mathrm{H}, \mathrm{s}), 0.81(3 \mathrm{H}, \mathrm{s}), 1.10(3 \mathrm{H}, \mathrm{d}$, $J Z 7.2 \mathrm{~Hz}), 1.30(3 \mathrm{H}, \mathrm{s}), 1.58(1 \mathrm{H}, \mathrm{dd}, J Z 0.0,13.8 \mathrm{~Hz})$, $1.96(1 \mathrm{H}, \mathrm{m}), 2.46(1 \mathrm{H}, \mathrm{dddd}, J Z 7.0,7.0,7.0,7.0 \mathrm{~Hz}), 2.56$ $(1 \mathrm{H}, \mathrm{dd}, J Z 5.7,13.8 \mathrm{~Hz}), 3.11$ (1H, dddd, $J Z 4.5,4.5,10.7$, $10.7 \mathrm{~Hz}), 3.31(1 \mathrm{H}, \mathrm{d}, J Z 9.0 \mathrm{~Hz}), 3.33(3 \mathrm{H}, \mathrm{s}), 3.49(1 \mathrm{H}, \mathrm{d}$, $J Z 9.0 \mathrm{~Hz}), 4.21(1 \mathrm{H}, \mathrm{d}, J Z 5.7 \mathrm{~Hz}), 4.53(1 \mathrm{H}, \mathrm{ddd}, J Z 5.6$, 7.5, 7.5 Hz); ${ }^{13} \mathrm{C}$ NMR $12.3\left(\mathrm{CH}_{3}\right), 16.0\left(\mathrm{CH}_{3}\right), 17.0\left(\mathrm{CH}_{3}\right)$, $20.9\left(\mathrm{CH}_{2}\right), 25.2\left(\mathrm{CH}_{3}\right), 27.9\left(\mathrm{CH}_{2}\right), 28.7\left(\mathrm{CH}_{2}\right), 31.9\left(\mathrm{CH}_{2}\right)$, $32.2\left(\mathrm{CH}_{2}\right), 34.3\left(\mathrm{CH}_{2}\right), 35.2(\mathrm{CH}), 35.5(\mathrm{CH}), 35.9(\mathrm{C})$, $36.9\left(\mathrm{CH}_{2}\right), 39.5\left(2 ! \mathrm{CH}_{2}\right), 41.1(\mathrm{C}), 44.7(\mathrm{CH}), 54.4(\mathrm{CH})$, 
$55.5\left(\mathrm{CH}_{3}\right), 56.2(\mathrm{CH}), 63.5(\mathrm{CH}), 68.5\left(\mathrm{CH}_{2}\right), 78.9(\mathrm{CH})$, $79.8(\mathrm{CH}), 81.8(\mathrm{CH}), 85.3(\mathrm{C}), 120.3(\mathrm{C}) ; \mathrm{MS} \mathrm{m} / \mathrm{z}(\mathrm{rel}$ intensity) $462\left(\mathrm{M}^{\mathrm{C}}, ! 1\right), 444$ (1), 431 (28), 287 (100); HRMS calcd for $\mathrm{C}_{28} \mathrm{H}_{46} \mathrm{O}_{5} 462.3345$; found 462.3338 . Anal. Calcd for $\mathrm{C}_{28} \mathrm{H}_{46} \mathrm{O}_{5}$ : C, 72.69; H, 10.02. Found: C, $72.83 ; \mathrm{H}, 9.78$.

\subsection{4. (22S,23R,25S)-3b-Methoxy-5a-spirostan-} 23,25- diol (19). A solution of compound $18(10 \mathrm{mg}$, $0.02 \mathrm{mmol})$ in $\mathrm{CHCl}_{3}(10 \mathrm{~mL})$ was treated with an undetermined catalytic amount of $\mathrm{HCl}$ (some gas taken with a Pasteur pipet from of a concd $\mathrm{HCl}$ bottle) and stirred at room temperature for $24 \mathrm{~h}$. The reaction mixture was poured into aqueous saturated $\mathrm{NaHCO}_{3}$ and extracted with $\mathrm{CHCl}_{3}$. Chromatotron chromatography (hexanes-EtOAc, $85: 15)$ of the residue afforded compound $19(5 \mathrm{mg}, 0.01$ mmol, 50\%): $\mathrm{mp} 250.5-252.58 \mathrm{C}$ (from EtOAc- $n$-hexane); $[\mathrm{a}]_{\mathrm{D}} \mathrm{K} 80$ (c 0.45$)$; IR 3601, $3492 \mathrm{~cm}^{\mathrm{K} 1}$; ${ }^{1} \mathrm{H}$ NMR 0.63 $(1 \mathrm{H}, \mathrm{m}), 0.77(3 \mathrm{H}, \mathrm{s}), 0.80(3 \mathrm{H}, \mathrm{s}), 1.12(3 \mathrm{H}, \mathrm{s}), 1.15(3 \mathrm{H}$, d, $J Z 6.9 \mathrm{~Hz}), 1.82(1 \mathrm{H}$, ddd, $J Z 2.8,2.8,14.3 \mathrm{~Hz}), 1.96$ $(1 \mathrm{H}, \mathrm{dd}, J Z 3.2,14.3 \mathrm{~Hz}), 2.30(1 \mathrm{H}$, dddd, JZ6.9, 6.9, $6.9,6.9 \mathrm{~Hz}), 3.11$ (1H, dddd, $J Z 4.6,4.6,10.9,10.9 \mathrm{~Hz})$, $3.33(3 \mathrm{H}, \mathrm{s}), 3.39(1 \mathrm{H}, \mathrm{dd}, J Z 2.8,11.8 \mathrm{~Hz}), 3.63(1 \mathrm{H}, \mathrm{dd}$, $J Z 2.8,2.8 \mathrm{~Hz}), 3.77(1 \mathrm{H}, \mathrm{d}, J Z 11.8 \mathrm{~Hz}), 4.47(1 \mathrm{H}, \mathrm{ddd}$, $J Z 5.7,7.8,7.8 \mathrm{~Hz}) ;{ }^{13} \mathrm{C}$ NMR $12.3\left(\mathrm{CH}_{3}\right), 16.2\left(\mathrm{CH}_{3}\right)$, $16.7\left(\mathrm{CH}_{3}\right), 20.9 \quad\left(\mathrm{CH}_{2}\right), 26.2\left(\mathrm{CH}_{3}\right), 27.8\left(\mathrm{CH}_{2}\right), 28.7$ $\left(\mathrm{CH}_{2}\right), 32.0\left(\mathrm{CH}_{2}\right), 32.2\left(\mathrm{CH}_{2}\right), 34.3\left(\mathrm{CH}_{2}\right), 35.2(\mathrm{CH}), 35.9$ (C), $36.9\left(\mathrm{CH}_{2}\right), 38.3\left(\mathrm{CH}_{2}\right), 39.6\left(\mathrm{CH}_{2}\right), 40.4(\mathrm{CH}), 41.0$ (C), $44.8(\mathrm{CH}), 54.4(\mathrm{CH}), 55.5\left(\mathrm{CH}_{3}\right), 56.4(\mathrm{CH}), 64.1$ $(\mathrm{CH}), 68.8(\mathrm{C}), 69.2\left(\mathrm{CH}_{2}\right), 70.9(\mathrm{CH}), 79.8(\mathrm{CH}), 81.8$ $(\mathrm{CH}), 108.7(\mathrm{C}) ; \mathrm{MS} \mathrm{m} / \mathrm{z}$ (rel intensity) $462\left(\mathrm{M}^{\mathrm{C}}, 2\right), 444$ (2), 426 (2), 411 (2), 361 (46), 287 (100); HRMS calcd for $\mathrm{C}_{28} \mathrm{H}_{46} \mathrm{O}_{5}$ 462.3345; found 462.3401. Anal. Calcd for $\mathrm{C}_{28} \mathrm{H}_{46} \mathrm{O}_{5}: \mathrm{C}, 72.69 ; \mathrm{H}, 10.02$. Found: C, 72.71; H, 10.13 .

4.1.5. $\quad(22 S, 23 R, 25 R)$-3b-Methoxy-23-tertbutyldimethyl- silyloxy-26-acetoxy-22,25-epoxy$5 \mathrm{a}$-furostan (20) and $(22 R, 23 R, 25 R)-3 \mathrm{~b}-$ methoxy-23-tert-butyldimethylsilyl- oxy-26acetoxy-22,25-epoxy-5a-furostan (21). A solution of the alcohol 14- $R(137 \mathrm{mg}, 0.22 \mathrm{mmol})$ in cyclohexane $(25$ $\mathrm{mL}$ ) containing (diacetoxyiodo)benzene $(84 \mathrm{mg}$,

$0.264 \mathrm{mmol}$ ) and iodine (56 $\mathrm{mg}, 0.22 \mathrm{mmol}$ ) was irradiated with two $80 \mathrm{~W}$ tungsten-filament lamps at $558 \mathrm{C}$ for $7 \mathrm{~h}$. The reaction mixture was then poured into aqueous solution of sodium thiosulfate $(10 \%)$ and extracted with $\mathrm{Et}_{2} \mathrm{O}$. The combined organic extracts were washed with brine, dried $\left(\mathrm{Na}_{2} \mathrm{SO}_{4}\right)$ and concentrated. Chromatotron chromatography (hexanes-EtOAc, 95:5) of the residue afforded compound $20(38 \mathrm{mg}, 0.061 \mathrm{mmol}, 27.8 \%)$ and compound 21 ( $76 \mathrm{mg}$,

0.122 mmol, 55.3\%). Compound 20: amorphous; $[\mathrm{a}]_{\mathrm{D}}$ K27 (c 0.064); IR $1746 \mathrm{~cm}^{\mathrm{K} 1}$; ${ }^{1} \mathrm{H}$ NMR $0.07(3 \mathrm{H}, \mathrm{s})$, $0.10(3 \mathrm{H}$

s), $0.63(1 \mathrm{H}, \mathrm{m}), 0.80(3 \mathrm{H}, \mathrm{s}), 0.85(3 \mathrm{H}, \mathrm{s}), 0.90(9 \mathrm{H}, \mathrm{s}), 1.06$ $(3 \mathrm{H}, \mathrm{d}, J Z 7.3 \mathrm{~Hz}), 1.20(3 \mathrm{H}, \mathrm{s}), 1.85(1 \mathrm{H}, \mathrm{dd}, J Z 7.5$, $11.1 \mathrm{~Hz}), 1.90(1 \mathrm{H}, \mathrm{ddd}, J Z 6.0,6.8,12.3 \mathrm{~Hz}), 1.98(1 \mathrm{H}, \mathrm{dd}$, $J Z 11.2,10.4 \mathrm{~Hz}), 2.05(3 \mathrm{H}, \mathrm{s}), 2.31$ (1H, dddd, $J Z 6.9,6.9$, $6.9,6.9 \mathrm{~Hz}), 3.10(1 \mathrm{H}$, dddd, $J Z 4.6,4.6,10.7,10.7 \mathrm{~Hz})$, $3.35(3 \mathrm{H}, \mathrm{s}), 4.01(1 \mathrm{H}, \mathrm{d}, J Z 10.9 \mathrm{~Hz}), 4.10(1 \mathrm{H}, \mathrm{d}, J \mathrm{Z}$ $10.9 \mathrm{~Hz}), 4.22(1 \mathrm{H}, \mathrm{dd}, J Z 7.5,10.4 \mathrm{~Hz}), 4.59(1 \mathrm{H}$, ddd, $J Z$ 6.8, 6.8, 6.8 Hz); ${ }^{13} \mathrm{C}$ NMR K5.3 $\left(\mathrm{CH}_{3}\right), \mathrm{K} 4.0\left(\mathrm{CH}_{3}\right), 12.3$ $\left(\mathrm{CH}_{3}\right), 16.0\left(\mathrm{CH}_{3}\right), 16.5\left(\mathrm{CH}_{3}\right), 17.8(\mathrm{C}), 21.0\left(\mathrm{CH}_{2}\right), 21.0$ $\left(\mathrm{CH}_{3}\right), 25.0\left(\mathrm{CH}_{3}\right), 25.8\left(3 ! \mathrm{CH}_{3}\right), 27.9\left(\mathrm{CH}_{2}\right), 28.8\left(\mathrm{CH}_{2}\right)$, $32.3\left(\mathrm{CH}_{2}\right), 33.4\left(\mathrm{CH}_{2}\right), 34.3\left(\mathrm{CH}_{2}\right), 35.0(\mathrm{CH}), 35.9(\mathrm{C})$, $36.9\left(\mathrm{CH}_{2}\right), 37.4(\mathrm{CH}), 40.1\left(\mathrm{CH}_{2}\right), 40.2\left(\mathrm{CH}_{2}\right), 41.2(\mathrm{C})$,
$44.8(\mathrm{CH}), 54.5(\mathrm{CH}), 55.5\left(\mathrm{CH}_{3}\right), 55.6(\mathrm{CH}), 61.9(\mathrm{CH})$, $71.0\left(\mathrm{CH}_{2}\right), 72.7(\mathrm{CH}), 78.5(\mathrm{C}), 79.8(\mathrm{CH}), 81.8(\mathrm{CH})$, $118.0(\mathrm{C}), 170.9(\mathrm{C})$; MS m/z (rel intensity) $618\left(\mathrm{M}^{\mathrm{C}}, ! 1\right.$ ), 617 (!1), 561 (6), 475 (31), 287 (20); HRMS calcd for $\mathrm{C}_{36} \mathrm{H}_{62} \mathrm{O}_{6} \mathrm{Si}$ 618.4316; found 618.4335. Anal. Calcd for $\mathrm{C}_{36} \mathrm{H}_{62} \mathrm{O}_{6} \mathrm{Si}: \mathrm{C}, 69.86 ; \mathrm{H}, 10.10$. Found: C, 69.98; H, 10.22. Compound 21: amorphous; [a] $]_{\mathrm{D}} \mathrm{K} 58.2$ ( $c$ 0.5); IR 1745 $\mathrm{cm}^{\mathrm{K} 1}$; ${ }^{1} \mathrm{H}$ NMR $0.06(3 \mathrm{H}, \mathrm{s}), 0.07(3 \mathrm{H}, \mathrm{s}), 0.63(1 \mathrm{H}, \mathrm{m})$, $0.76(3 \mathrm{H}, \mathrm{s}), 0.80(3 \mathrm{H}, \mathrm{s}), 0.89(9 \mathrm{H}, \mathrm{s}), 1.02(3 \mathrm{H}, \mathrm{d}, J \mathrm{Z}$ $7.0 \mathrm{~Hz}), 1.29(3 \mathrm{H}, \mathrm{s}), 1.84(1 \mathrm{H}, \mathrm{dd}, J Z 0.0,13.5 \mathrm{~Hz}), 1.96$ $(1 \mathrm{H}$, ddd, $J Z 5.7,7.3,12.7 \mathrm{~Hz}), 2.04(1 \mathrm{H}, \mathrm{dd}, J Z 5.1,13.5$ Hz), 2.06 (3H, s), 2.33 (1H, dddd, JZ6.9, 6.9, 6.9, $6.9 \mathrm{~Hz})$, $3.11(1 \mathrm{H}$, dddd, $J Z 4.5,4.5,10.9,10.9 \mathrm{~Hz}), 3.33(3 \mathrm{H}, \mathrm{s})$, $4.04(1 \mathrm{H}, \mathrm{d}, J Z 10.6 \mathrm{~Hz}), 4.08(1 \mathrm{H}, \mathrm{d}, J Z 10.6 \mathrm{~Hz}), 4.17$ $(1 \mathrm{H}, \mathrm{d}, J Z 4.4 \mathrm{~Hz}), 4.52(1 \mathrm{H}, \mathrm{ddd}, J Z 5.7,7.9,7.9 \mathrm{~Hz}) ;{ }^{13} \mathrm{C}$ NMR K5.1 $\left(\mathrm{CH}_{3}\right), \mathrm{K} 5.0\left(\mathrm{CH}_{3}\right), 12.3\left(\mathrm{CH}_{3}\right), 16.4\left(\mathrm{CH}_{3}\right)$, $16.8\left(\mathrm{CH}_{3}\right), 17.9(\mathrm{C}), 20.91\left(\mathrm{CH}_{2}\right), 20.96\left(\mathrm{CH}_{3}\right), 25.7$ $\left(3 ! \mathrm{CH}_{3}\right), 26.1\left(\mathrm{CH}_{3}\right), 27.9\left(\mathrm{CH}_{2}\right), 28.7\left(\mathrm{CH}_{2}\right), 32.1\left(\mathrm{CH}_{2}\right)$, $32.2\left(\mathrm{CH}_{2}\right), 34.3\left(\mathrm{CH}_{2}\right), 35.3(\mathrm{CH}), 35.9(\mathrm{C}), 36.4(\mathrm{CH})$, $36.9\left(\mathrm{CH}_{2}\right), 39.8\left(\mathrm{CH}_{2}\right), 41.1(\mathrm{C}), 42.7\left(\mathrm{CH}_{2}\right), 44.8(\mathrm{CH})$, $54.4(\mathrm{CH}), 55.5\left(\mathrm{CH}_{3}\right), 56.3(\mathrm{CH}), 63.2(\mathrm{CH}), 70.4\left(\mathrm{CH}_{2}\right)$, $78.7(\mathrm{CH}), 79.8(\mathrm{CH}), 81.4(\mathrm{CH}), 81.8(\mathrm{C}), 122.0(\mathrm{C}), 170.9$ (C); MS $m / z$ (rel intensity) $618\left(\mathrm{M}^{\mathrm{C}}, 1\right), 603(! 1), 561$ (43), 545 (23), 287 (68); HRMS calcd for $\mathrm{C}_{36} \mathrm{H}_{62} \mathrm{O}_{6} \mathrm{Si}$ 618.4316; found 618.4324. Anal. Calcd for $\mathrm{C}_{36} \mathrm{H}_{62} \mathrm{O}_{6} \mathrm{Si}: \mathrm{C}$, 69.86; H, 10.10. Found: C, 69.71; H, 9.98.

4.1.6. $(22 S, 23 R, 25 R)$-3b-Methoxy-22,25-epoxy-5afuro- stan-23,26-diol (22). To a solution of compound 20 (38 mg, $0.061 \mathrm{mmol}$ ) in THF ( $8 \mathrm{~mL}$ ) was added TBAF $(0.4 \mathrm{~mL}, 0.4 \mathrm{mmol}, 1.0 \mathrm{M}$ in THF) and stirred at room temperature for $1.5 \mathrm{~h}$. The mixture was then poured into aqueous saturated solution of $\mathrm{NaHCO}_{3}$ and extracted with $\mathrm{Et}_{2} \mathrm{O}$. The organic extracts were washed with brine, dried $\left(\mathrm{Na}_{2} \mathrm{SO}_{4}\right)$ and concentrated. Chromatotron chromatography (benzene-EtOAc, 90:10) of the residue afforded $(22 S, 23 R, 25 R)$-3b-methoxy-26-acetoxy-22,25-epoxy-5afurostan-23-ol (28 mg, $0.055 \mathrm{mmol}, 90 \%)$ : amorphous; [a $]_{\mathrm{D}}$ C 10 (c 0.39); IR 3443, $1745 \mathrm{~cm}^{\mathrm{K} 1}$; ${ }^{1} \mathrm{H}$ NMR $0.62(1 \mathrm{H}, \mathrm{m})$, $0.80(3 \mathrm{H}, \mathrm{s}), 0.89(3 \mathrm{H}, \mathrm{s}), 1.12(3 \mathrm{H}, \mathrm{d}, J Z 7.5 \mathrm{~Hz}), 1.19(3 \mathrm{H}$, s), $1.82(1 \mathrm{H}, \mathrm{dd}, J Z 9.1,12.4 \mathrm{~Hz}), 1.87(1 \mathrm{H}, \mathrm{ddd}, J Z 5.7$, 7.3, $12.2 \mathrm{~Hz}), 2.06(3 \mathrm{H}, \mathrm{s}), 2.36$ (1H, dddd, JZ3.5, 7.4, 7.4, $7.4 \mathrm{~Hz}), 3.11(1 \mathrm{H}$, dddd, $J Z 4.5,4.5,10.6,10.6, \mathrm{~Hz}), 3.33$ $(3 \mathrm{H}, \mathrm{s}), 3.96(1 \mathrm{H}, \mathrm{d}, J Z 10.8 \mathrm{~Hz}), 4.06(1 \mathrm{H}, \mathrm{d}, J Z 10.8 \mathrm{~Hz})$, $4.25(1 \mathrm{H}$, ddd, $J Z 8.8,8.8,8.8 \mathrm{~Hz}), 4.54(1 \mathrm{H}$, ddd, $J Z 7.0$, 7.0, 7.0 Hz); ${ }^{13} \mathrm{C}$ NMR $12.2\left(\mathrm{CH}_{3}\right), 15.9\left(\mathrm{CH}_{3}\right), 17.3\left(\mathrm{CH}_{3}\right)$, $20.9\left(\mathrm{CH}_{3}\right), 20.9\left(\mathrm{CH}_{2}\right), 25.2\left(\mathrm{CH}_{3}\right), 27.8\left(\mathrm{CH}_{2}\right), 28.7\left(\mathrm{CH}_{2}\right)$, $32.3\left(\mathrm{CH}_{2}\right), 32.8\left(\mathrm{CH}_{2}\right), 34.2\left(\mathrm{CH}_{2}\right), 34.7(\mathrm{CH}), 35.9(\mathrm{C})$, $36.8\left(\mathrm{CH}_{2}\right), 39.1(\mathrm{CH}), 40.0\left(\mathrm{CH}_{2}\right), 41.4(\mathrm{C}), 41.7\left(\mathrm{CH}_{2}\right)$, $44.7(\mathrm{CH}), 54.5(\mathrm{CH}), 55.5(\mathrm{CH}), 55.5\left(\mathrm{CH}_{3}\right), 63.3(\mathrm{CH})$, $70.4\left(\mathrm{CH}_{2}\right), 72.4(\mathrm{CH}), 78.5(\mathrm{C}), 79.7(\mathrm{CH}), 83.7(\mathrm{CH})$, $128.3(\mathrm{C}), 170.8(\mathrm{C}) ; \mathrm{MS} m / z$ (rel intensity) $505\left(\mathrm{M}^{\mathrm{C}} \mathrm{C} \mathrm{H}\right.$, ! 1), 486 (! 1), 471 (!1), 413 ! 1), 361 (83), 287 (100); HRMS calcd for $\mathrm{C}_{30} \mathrm{H}_{46} \mathrm{O}_{5}$ 486.3345; found 486.3335. Anal. Calcd for $\mathrm{C}_{30} \mathrm{H}_{48} \mathrm{O}_{6}$ : C, 71.39; H, 9.59. Found: C, $71.52 ; \mathrm{H}, 9.71$. A solution of this acetate $(24 \mathrm{mg}$, $0.047 \mathrm{mmol})$ in $\mathrm{MeOH}(15 \mathrm{~mL})$ containing $\mathrm{KOH}(0.3 \mathrm{~g})$ was stirred at room temperature for $8 \mathrm{~h}$. The mixture was poured into water and extracted with AcOEt. The combined extracts were washed with brine, dried $\left(\mathrm{Na}_{2} \mathrm{SO}_{4}\right)$ and concentrated. Chromatotron chromatography (hexanes-EtOAc, 7:3) of the residue afforded compound $22(18 \mathrm{mg}, 0.039 \mathrm{mmol}$, 82\%): mp 166.5-168 8C (from EtOAc- $n$-hexane); $[\mathrm{a}]_{\mathrm{D}}$ $\mathrm{K} 4$ 
(c 0.55); IR $3408 \mathrm{~cm}^{\mathrm{K} 1} ;{ }^{1} \mathrm{H}$ NMR $0.62(1 \mathrm{H}, \mathrm{m}), 0.80(3 \mathrm{H}$, s), $0.89(3 \mathrm{H}, \mathrm{s}), 1.13(3 \mathrm{H}, \mathrm{s}), 1.16(3 \mathrm{H}, \mathrm{d}, J \mathrm{Z} 7.5 \mathrm{~Hz}), 1.96$ $(1 \mathrm{H}, \mathrm{dd}, J Z 8.1,12.3 \mathrm{~Hz}), 2.04(1 \mathrm{H}$, ddd, JZ7.3, 7.3, $7.3 \mathrm{~Hz}), 2.15(1 \mathrm{H}, \mathrm{dd}, J Z 9.8,12.4 \mathrm{~Hz}), 2.39$ (1H, dddd, $J$ Z $3.3,7.3,7.3,7.3 \mathrm{~Hz}), 3.11(1 \mathrm{H}$, dddd, $J Z 4.5,4.5,10.7$, $10.7 \mathrm{~Hz}), 3.25(1 \mathrm{H}, \mathrm{d}, J Z 11.4 \mathrm{~Hz}), 3.33(3 \mathrm{H}, \mathrm{s}), 3.45(1 \mathrm{H}$, d, $J Z 11.5 \mathrm{~Hz}), 4.26(1 \mathrm{H}, \mathrm{dd}, J Z 9.0,9.0 \mathrm{~Hz}), 4.58(1 \mathrm{H}$, ddd, $J Z 7.3,7.3,7.3 \mathrm{~Hz}) ;{ }^{13} \mathrm{C}$ NMR $12.2\left(\mathrm{CH}_{3}\right), 16.1\left(\mathrm{CH}_{3}\right)$, 17.2 $\left(\mathrm{CH}_{3}\right), 20.8\left(\mathrm{CH}_{2}\right), 24.9\left(\mathrm{CH}_{3}\right), 27.8\left(\mathrm{CH}_{2}\right), 28.7\left(\mathrm{CH}_{2}\right)$, $32.2\left(\mathrm{CH}_{2}\right), 34.0\left(\mathrm{CH}_{2}\right), 34.2\left(\mathrm{CH}_{2}\right), 34.8(\mathrm{CH}), 35.9(\mathrm{C})$, $36.9\left(\mathrm{CH}_{2}\right), 38.5(\mathrm{CH}), 38.5\left(\mathrm{CH}_{2}\right), 39.7\left(\mathrm{CH}_{2}\right), 41.5(\mathrm{C})$, $44.7(\mathrm{CH}), 54.4(\mathrm{CH}), 55.4\left(\mathrm{CH}_{3}\right), 55.6(\mathrm{CH}), 63.8(\mathrm{CH})$, $68.1\left(\mathrm{CH}_{2}\right), 73.4(\mathrm{CH}), 79.7(\mathrm{CH}), 81.7(\mathrm{C}), 83.8(\mathrm{CH})$, 118.2 (C); $\mathrm{MS} \mathrm{m} / \mathrm{z}$ (rel intensity) $444\left(\mathrm{M}^{\mathrm{C}} \mathrm{KH}_{2} \mathrm{O}, 2\right), 431$ (6), 426 (9), 411 (4), 287 (100); HRMS calcd for $\mathrm{C}_{28} \mathrm{H}_{44} \mathrm{O}_{4}$ 444.3240; found 444.3221. Anal. Calcd for $\mathrm{C}_{28} \mathrm{H}_{46} \mathrm{O}_{5}$ : C, 72.69; H, 10.02. Found: C, 72.71; H, 10.14.

4.1.7. $(22 R, 23 R, 25 R)$-3b-Methoxy-22,25-epoxy-5afuro- stan-23,26-diol (23). To a solution of compound $21(23 \mathrm{mg}$,

$0.037 \mathrm{mmol})$ in THF $(5 \mathrm{~mL})$ was added TBAF $(0.2 \mathrm{~mL}$, $0.2 \mathrm{mmol}, 1.0 \mathrm{M}$ in THF) and stirred at room temperature for $3 \mathrm{~h}$. The mixture was then poured into aqueous saturated solution of $\mathrm{NaHCO}_{3}$ and extracted with $\mathrm{Et}_{2} \mathrm{O}$. The organic extracts were washed with brine, dried $\left(\mathrm{Na}_{2} \mathrm{SO}_{4}\right)$ and concentrated. The crude residue was saponified with methanolic $\mathrm{KOH}(10 \mathrm{~mL}, 3 \%)$ for $4 \mathrm{~h}$ at room temperature. Chromatotron chromatography (benzene-EtOAc, 75:25) of the residue afforded compound $23(12 \mathrm{mg}, 0.026 \mathrm{mmol}, 70 \%)$ : mp 269.5-270 8C (from MeOH); [a $]_{\mathrm{D}} \mathrm{K} 72$ (c 0.122); IR $3317 \mathrm{~cm}^{\mathrm{K} 1} ;{ }^{1} \mathrm{H}$ NMR $0.63(1 \mathrm{H}, \mathrm{m}), 0.79(3 \mathrm{H}, \mathrm{s}), 0.80(3 \mathrm{H}$, s), $1.11(3 \mathrm{H}, \mathrm{d}, J Z 6.7 \mathrm{~Hz}), 1.27(3 \mathrm{H}, \mathrm{s}), 1.93(1 \mathrm{H}, \mathrm{dd}, J \mathrm{Z}$ $0.0,14.0 \mathrm{~Hz}), 1.96(1 \mathrm{H}, \mathrm{ddd}, J Z 5.8,7.4,12.4 \mathrm{~Hz}), 2.28$ $(1 \mathrm{H}, \mathrm{dd}, J Z 5.5,14.0 \mathrm{~Hz}), 2.53(1 \mathrm{H}$, dddd, $J Z 7.0,7.0,7.0$, $7.0 \mathrm{~Hz}), 3.11(1 \mathrm{H}$, dddd, $J Z 4.5,4.5,10.7,10.7 \mathrm{~Hz}), 3.33$ $(3 \mathrm{H}, \mathrm{s}), 3.39(1 \mathrm{H}, \mathrm{d}, J Z 11.2 \mathrm{~Hz}), 3.65(1 \mathrm{H}, \mathrm{d}, J Z 11.2 \mathrm{~Hz})$, $4.02(1 \mathrm{H}, \mathrm{d}, J Z 5.5 \mathrm{~Hz}), 4.51(1 \mathrm{H}$, ddd, $J Z 5.7,7.9,7.9 \mathrm{~Hz})$; ${ }^{13} \mathrm{C}$ NMR $12.3\left(\mathrm{CH}_{3}\right), 16.1\left(\mathrm{CH}_{3}\right), 17.1\left(\mathrm{CH}_{3}\right), 20.9\left(\mathrm{CH}_{2}\right)$, $26.2\left(\mathrm{CH}_{3}\right), 27.8\left(\mathrm{CH}_{2}\right), 28.7\left(\mathrm{CH}_{2}\right), 32.0\left(\mathrm{CH}_{2}\right), 32.2\left(\mathrm{CH}_{2}\right)$, $34.3\left(\mathrm{CH}_{2}\right), 35.1(\mathrm{CH}), 35.9(\mathrm{C}), 36.3(\mathrm{CH}), 36.9\left(\mathrm{CH}_{2}\right)$, $39.8\left(\mathrm{CH}_{2}\right), 41.1(\mathrm{C}), 42.4\left(\mathrm{CH}_{2}\right), 44.8(\mathrm{CH}), 54.4(\mathrm{CH})$, $55.5\left(\mathrm{CH}_{3}\right), 56.3(\mathrm{CH}), 63.6(\mathrm{CH}), 68.9\left(\mathrm{CH}_{2}\right), 77.0(\mathrm{CH})$, $79.8(\mathrm{CH}), 81.0(\mathrm{CH}), 83.2(\mathrm{C}), 120.6(\mathrm{C}) ; \mathrm{MS} \mathrm{m} / \mathrm{z}(\mathrm{rel}$ intensity) $444\left(\mathrm{M}^{\mathrm{C}} \mathrm{KH}_{2} \mathrm{O}, ! 1\right), 413$ (2), 287 (45); HRMS calcd for $\mathrm{C}_{28} \mathrm{H}_{44} \mathrm{O}_{4} 444.3240$; found 444.3268. Anal. Calcd for $\mathrm{C}_{28} \mathrm{H}_{46} \mathrm{O}_{5}: \mathrm{C}, 72.69 ; \mathrm{H}, 10.02$. Found: $\mathrm{C}, 72.69$; $\mathrm{H}, 9.90$.

4.1.8.

$(22 S, 23 S, 25 S)$-3b-Methoxy-23-tertbutyldimethyl- silyloxy-26-acetoxy-22,25-epoxy$5 a-f u r o s t a n(25)$ and $(22 R, 23 S, 25 S)$-3b-methoxy23-tert-butyldimethylsilyl- oxy-26-acetoxy-22,25epoxy-5a-furostan (26). A solution of the alcohol 12-S (420 mg, $0.68 \mathrm{mmol})$ in cyclohexane $(70 \mathrm{~mL})$ containing (diacetoxyiodo)benzene (437 mg,

$1.36 \mathrm{mmol})$ and iodine $(172 \mathrm{mg}, 0.68 \mathrm{mmol})$ was irradiated with a $80 \mathrm{~W}$ tungsten-filament lamp at $708 \mathrm{C}$ for $1 \mathrm{~h}$. The reaction mixture was then poured into aqueous solution of sodium thiosulfate $(10 \%)$ and extracted with $\mathrm{CH}_{2} \mathrm{Cl}_{2}$. The combined organic extracts were washed with brine, dried $\left(\mathrm{Na}_{2} \mathrm{SO}_{4}\right)$ and concentrated. Flash-chromatography (hexanesEtOAc, 93:7) of the residue afforded compound 25 (96 $\mathrm{mg}$,

$0.15 \mathrm{mmol}, 23 \%)$ and compound $26(310 \mathrm{mg}, 0.50 \mathrm{mmol}$, 74\%). Compound 25: mp 62.4-63.7 8C (from EtOAc); $[a]_{D}$
C21.2 (c 0.08); IR $1745 \mathrm{~cm}^{\mathrm{K} 1} ;{ }^{1} \mathrm{H}$ NMR 0.07 (3H, s), 0.08 $(3 \mathrm{H}, \mathrm{s}), 0.64(1 \mathrm{H}, \mathrm{m}), 0.81(3 \mathrm{H}, \mathrm{s}), 0.86(3 \mathrm{H}, \mathrm{s}), 0.90(9 \mathrm{H}, \mathrm{s})$, $1.13(3 \mathrm{H}, \mathrm{d}, J Z 7.2 \mathrm{~Hz}), 1.33(3 \mathrm{H}, \mathrm{s}), 1.89(1 \mathrm{H}, \mathrm{dd}, J Z 3.3$, $13.1 \mathrm{~Hz}), 2.01(1 \mathrm{H}, \mathrm{dd}, J Z 5.2,13.1 \mathrm{~Hz}), 2.07(3 \mathrm{H}, \mathrm{s}), 3.11$ $(1 \mathrm{H}$, dddd, $J Z 4.7,4.7,11.0,11.0 \mathrm{~Hz}), 3.33(3 \mathrm{H}, \mathrm{s}), 4.02$ $(1 \mathrm{H}, \mathrm{d}, J Z 10.7 \mathrm{~Hz}), 4.11(1 \mathrm{H}, \mathrm{d}, J Z 10.4 \mathrm{~Hz}), 4.26(1 \mathrm{H}, \mathrm{dd}$, $J Z 3.4,5.1 \mathrm{~Hz}), 4.38(1 \mathrm{H}, \mathrm{ddd}, J Z 7.5,7.5,9.4 \mathrm{~Hz}) ; \mathrm{NOE}$ correlation from $23-\mathrm{H}$ to $21-\mathrm{Me}$ and to $27-\mathrm{Me} ;{ }^{13} \mathrm{C}$ NMR $(100.6 \mathrm{MHz}) \mathrm{K} 5.2\left(\mathrm{CH}_{3}\right), \mathrm{K} 3.8\left(\mathrm{CH}_{3}\right), 12.3\left(\mathrm{CH}_{3}\right), 16.2$ $\left(\mathrm{CH}_{3}\right), 17.0\left(\mathrm{CH}_{3}\right), 17.8(\mathrm{C}), 20.9\left(\mathrm{CH}_{3}\right), 21.2\left(\mathrm{CH}_{2}\right), 25.6$ $\left(\mathrm{CH}_{3}\right), 25.9\left(3 ! \mathrm{CH}_{3}\right), 27.9\left(\mathrm{CH}_{2}\right), 28.8\left(\mathrm{CH}_{2}\right), 32.5\left(\mathrm{CH}_{2}\right)$, $33.1\left(\mathrm{CH}_{2}\right), 34.3\left(\mathrm{CH}_{2}\right), 34.4(\mathrm{CH}), 36.0(\mathrm{C}), 36.9\left(\mathrm{CH}_{2}\right)$, $39.4(\mathrm{CH}), 41.0\left(\mathrm{CH}_{2}\right), 41.1(\mathrm{C}), 42.6\left(\mathrm{CH}_{2}\right), 44.9(\mathrm{CH})$, $54.6(\mathrm{CH}), 54.9(\mathrm{CH}), 55.5\left(\mathrm{CH}_{3}\right), 62.1(\mathrm{CH}), 70.5\left(\mathrm{CH}_{2}\right)$, $77.7(\mathrm{CH}), 79.8(\mathrm{CH}), 80.5(\mathrm{C}), 81.7(\mathrm{CH}), 120.6(\mathrm{C}), 170.8$ (C); MS $m / z$ (rel intensity) $618\left(\mathrm{M}^{\mathrm{C}}, ! 11\right), 561(3), 545(2)$, 198 (100); HRMS calcd for $\mathrm{C}_{36} \mathrm{H}_{62} \mathrm{O}_{6} \mathrm{Si}$ 618.4316; found 618.4317. Anal. Calcd for $\mathrm{C}_{36} \mathrm{H}_{62} \mathrm{O}_{6} \mathrm{Si}$ : C, 69.86; H, 10.10 . Found: C, 69.92; H, 10.04. Compound 26: mp 135.0 $136.08 \mathrm{C}$ (from EtOAc); [a] $]_{\mathrm{D}} \mathrm{K} 34$ (c 0.10 ); IR 1745 $\mathrm{cm}^{\mathrm{K}}{ }^{1} ;{ }^{1} \mathrm{H}$ NMR $0.06(3 \mathrm{H}, \mathrm{s}), 0.07(3 \mathrm{H}, \mathrm{s}), 0.60(1 \mathrm{H}, \mathrm{m})$, $0.77(3 \mathrm{H}, \mathrm{s}), 0.78(3 \mathrm{H}, \mathrm{s}), 0.88(9 \mathrm{H}, \mathrm{s}), 0.92(3 \mathrm{H}, \mathrm{d}, J Z 6.9$ $\mathrm{Hz}), 1.17(3 \mathrm{H}, \mathrm{s}), 1.89(1 \mathrm{H}, \mathrm{dd}, J Z 7.9,11.8 \mathrm{~Hz}), 2.05(3 \mathrm{H}$, s), $2.14(1 \mathrm{H}, \mathrm{m}), 3.10(1 \mathrm{H}$, dddd, JZ4.6, 4.6, 10.9, 10.9 $\mathrm{Hz}), 3.32(3 \mathrm{H}, \mathrm{s}), 3.97(1 \mathrm{H}, \mathrm{d}, J Z 10.8 \mathrm{~Hz}), 4.01(1 \mathrm{H}, \mathrm{dd}$, $J Z 7.8,10.6 \mathrm{~Hz}), 4.04(1 \mathrm{H}, \mathrm{d}, J Z 10.8 \mathrm{~Hz}), 4.43(1 \mathrm{H}, \mathrm{ddd}$, $J Z 7.4,7.4,7.4 \mathrm{~Hz}$ ); NOE correlation from $23-\mathrm{H}$ to $21-$ $\mathrm{Me}$ and to $27-\mathrm{Me} ;{ }^{13} \mathrm{C}$ NMR $(100.6 \mathrm{MHz}) \mathrm{K} 4.8\left(\mathrm{CH}_{3}\right)$, $\mathrm{K} 4.7\left(\mathrm{CH}_{3}\right), 12.3\left(\mathrm{CH}_{3}\right), 14.0\left(\mathrm{CH}_{3}\right), 16.6\left(\mathrm{CH}_{3}\right), 18.2$ (C), $20.9\left(\mathrm{CH}_{2}\right), 20.9\left(\mathrm{CH}_{3}\right), 25.1\left(\mathrm{CH}_{3}\right), 25.8\left(3\right.$ ! $\left.\mathrm{CH}_{3}\right)$, $27.9\left(\mathrm{CH}_{2}\right), 28.7 \quad\left(\mathrm{CH}_{2}\right), 31.8\left(\mathrm{CH}_{2}\right), 32.2\left(\mathrm{CH}_{2}\right), 34.3$ $\left(\mathrm{CH}_{2}\right), 34.9(\mathrm{CH}), 35.0(\mathrm{CH}), 35.8(\mathrm{C}), 36.9\left(\mathrm{CH}_{2}\right), 40.1$ $\left(\mathrm{CH}_{2}\right), 40.5\left(\mathrm{CH}_{2}\right), 40.9(\mathrm{C}), 44.7(\mathrm{CH}), 54.4(\mathrm{CH}), 55.5$ $\left(\mathrm{CH}_{3}\right), 56.2(\mathrm{CH}), \quad 61.4(\mathrm{CH}), 70.6(\mathrm{CH}), 71.1\left(\mathrm{CH}_{2}\right)$, 78.0 (C), $79.8(\mathrm{CH})$,

$80.8(\mathrm{CH}), 116.7(\mathrm{C}), 170.8(\mathrm{C}) ; \mathrm{MS} \mathrm{m} / \mathrm{z}$ (rel intensity) $618\left(\mathrm{M}^{\mathrm{C}}, ! 1\right.$ ) , $562(3), 545$ (2), 287 (100); HRMS calcd for $\mathrm{C}_{36} \mathrm{H}_{62} \mathrm{O}_{6} \mathrm{Si}$ 618.4316; found 618.4317. Anal. Calcd for $\mathrm{C}_{36} \mathrm{H}_{62} \mathrm{O}_{6} \mathrm{Si}: \mathrm{C}, 69.86 ; \mathrm{H}, 10.10$. Found: C, 69.84; H, 10.26 .

4.1.9. (22S,23S,25S)-3b-Methoxy-22,25-epoxy-5afuro- stan-23,26-diol (27). To a solution of compound 25 (96 mg,

$0.15 \mathrm{mmol})$ in THF $(22 \mathrm{~mL})$ was added TBAF $(0.75 \mathrm{~mL}$, $0.75 \mathrm{mmol}, 1.0 \mathrm{M}$ in THF) and stirred at room temperature for $2 \mathrm{~h}$. The mixture was then poured into aqueous saturated solution of $\mathrm{NaHCO}_{3}$ and extracted with $\mathrm{CH}_{2} \mathrm{Cl}_{2}$. The organic extracts were washed with brine, dried $\left(\mathrm{Na}_{2} \mathrm{SO}_{4}\right)$ and concentrated. A solution of the residue in $\mathrm{MeOH}$ (46 $\mathrm{mL})$ containing $\mathrm{KOH}(1.5 \mathrm{~g})$ was stirred at room temperature for $0.5 \mathrm{~h}$. The mixture was poured into water and extracted with EtOAc. The combined extracts were washed with brine, dried $\left(\mathrm{Na}_{2} \mathrm{SO}_{4}\right)$ and concentrated. Chromatotron chromatography (hexanes-EtOAc, 7:3) afforded compound 27 (48 mg, $0.10 \mathrm{mmol}, 67 \%)$ : $\mathrm{mp}$ 172.5-173.2 8C (from $n$-hexane-EtOAc); [a] $]_{\mathrm{D}} \mathrm{C} 30.8$ ( $c$ 0.12); IR $3352 \mathrm{~cm}^{\mathrm{K} 1} ;{ }^{1} \mathrm{H}$ NMR $0.62(1 \mathrm{H}, \mathrm{m}), 0.79(3 \mathrm{H}, \mathrm{s})$, $0.94(3 \mathrm{H}, \mathrm{s}), 1.19(3 \mathrm{H}, \mathrm{d}, J \mathrm{Z7} .3 \mathrm{~Hz}), 1.25(3 \mathrm{H}, \mathrm{s}), 1.89(1 \mathrm{H}$, dd, $J Z 0.0,13.6 \mathrm{~Hz}), 2.19(1 \mathrm{H}, \mathrm{dd}, J Z 4.9,13.7 \mathrm{~Hz}), 2.36$ (1H, dddd, $J Z 2.0,7.2,7.2,7.2 \mathrm{~Hz}$ ), 3.11 (1H, dddd, $J Z 4.6$, 4.6, $11.0,11.0 \mathrm{~Hz}), 3.32(3 \mathrm{H}, \mathrm{s}), 3.33(1 \mathrm{H}, \mathrm{d}, J Z 11.2 \mathrm{~Hz})$, $3.53(1 \mathrm{H}, \mathrm{d}, J Z 11.2 \mathrm{~Hz}), 3.93(1 \mathrm{H}, \mathrm{dd}, J Z 0.0,4.8 \mathrm{~Hz})$, $4.54(1 \mathrm{H}$, ddd, $J Z 7.8,7.8,7.8 \mathrm{~Hz})$; NOE correlation from 23-H to $21-\mathrm{Me} ;{ }^{13} \mathrm{C}$ NMR $(100.6 \mathrm{MHz}) 12.2\left(\mathrm{CH}_{3}\right), 16.1$ 
$\left(\mathrm{CH}_{3}\right), 19.3\left(\mathrm{CH}_{3}\right), 21.1\left(\mathrm{CH}_{2}\right), 26.1\left(\mathrm{CH}_{3}\right), 27.8\left(\mathrm{CH}_{2}\right), 28.8$ $\left(\mathrm{CH}_{2}\right), 32.5\left(\mathrm{CH}_{2}\right), 33.7\left(2 ! \mathrm{CH}_{2}\right), 34.3(\mathrm{CH}), 35.9(\mathrm{C})$, $36.8\left(\mathrm{CH}_{2}\right), 38.6(\mathrm{CH}), 41.0\left(\mathrm{CH}_{2}\right), 41.4(\mathrm{C}), 42.2\left(\mathrm{CH}_{2}\right)$, $44.8(\mathrm{CH}), 54.6(2 ! \mathrm{CH}), 55.5\left(\mathrm{CH}_{3}\right), 64.2(\mathrm{CH}), 68.3$ $\left(\mathrm{CH}_{2}\right), 74.8(\mathrm{CH}), 79.8(\mathrm{CH}), 82.4(\mathrm{C}), 83.8(\mathrm{CH}), 121.7$ (C); MS m/z (rel intensity) $462\left(\mathrm{M}^{\mathrm{C}}, ! 1\right), 444$ (7), 426 (16), 287 (100); HRMS calcd for $\mathrm{C}_{28} \mathrm{H}_{46} \mathrm{O}_{5}$ 462.3345; found 462.3326. Anal. Calcd for $\mathrm{C}_{28} \mathrm{H}_{46} \mathrm{O}_{5}$ : C, 72.69; H, 10.02 . Found: C, 72.77; H, 10.03 .

4.1.10. (22R,23S,25S)-3b-Methoxy-22,25-epoxy5a-furo- stan-23,26-diol (28). To a solution of compound $26(83 \mathrm{mg}$,

$0.13 \mathrm{mmol})$ in THF $(19 \mathrm{~mL})$ was added TBAF $(0.65 \mathrm{~mL}$, $0.65 \mathrm{mmol}, 1.0 \mathrm{M}$ in THF) and stirred at room temperature for $1 \mathrm{~h}$. The mixture was then poured into aqueous saturated solution of $\mathrm{NaHCO}_{3}$ and extracted with EtOAc. The organic extracts were washed with brine, dried $\left(\mathrm{Na}_{2} \mathrm{SO}_{4}\right)$ and concentrated. A solution of the residue in $\mathrm{MeOH}(40 \mathrm{~mL})$ containing $\mathrm{KOH}$ (1.3 g) was stirred at room temperature for $0.5 \mathrm{~h}$. The mixture was poured into water and extracted with EtOAc. The combined extracts were washed with brine, dried $\left(\mathrm{Na}_{2} \mathrm{SO}_{4}\right)$ and concentrated. Chromatotron chromatography (hexanes-EtOAc, 6:4) afforded compound 28

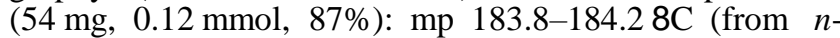
hexane-EtOAc); [a] $\mathrm{D}$ K46.7 (c 0.06); IR 3571, $3466 \mathrm{~cm}^{\mathrm{K} 1}$; ${ }^{1} \mathrm{H}$ NMR $0.63(1 \mathrm{H}, \mathrm{m}), 0.77(3 \mathrm{H}, \mathrm{s}), 0.78(3 \mathrm{H}, \mathrm{s}), 0.94(3 \mathrm{H}$, d, $J Z 7.0 \mathrm{~Hz}), 1.14(3 \mathrm{H}, \mathrm{s}), 1.97(1 \mathrm{H}, \mathrm{dd}, J Z 8.3,12.3 \mathrm{~Hz})$, $2.12(1 \mathrm{H}, \mathrm{dd}, J Z 10.5,12.4 \mathrm{~Hz}), 2.31(1 \mathrm{H}$, dddd, $J Z 6.9$, $6.9,6.9,6.9 \mathrm{~Hz}), 3.10$ (1H, dddd, JZ4.6, 4.6, 10.9, $10.9 \mathrm{~Hz}), 3.29(1 \mathrm{H}, \mathrm{d}, J Z 10.3 \mathrm{~Hz}), 3.32(3 \mathrm{H}, \mathrm{s}), 3.44$ $(1 \mathrm{H}, \mathrm{d}, J Z 11.3 \mathrm{~Hz}), 4.04(1 \mathrm{H}, \mathrm{m}), 4.55$ (1H, ddd, $J Z 7.1$, 7.1, 8.7 Hz); NOE correlation from $23-\mathrm{H}$ to $21-\mathrm{Me}$, to $20-\mathrm{H}$ and to $27-\mathrm{Me} ;{ }^{13} \mathrm{C}$ NMR $(100.6 \mathrm{MHz}) 12.2\left(\mathrm{CH}_{3}\right), 14.3$ $\left(\mathrm{CH}_{3}\right), 16.4\left(\mathrm{CH}_{3}\right), 20.9\left(\mathrm{CH}_{2}\right), 24.6\left(\mathrm{CH}_{3}\right), 27.8(\mathrm{CH}), 28.6$ $\left(\mathrm{CH}_{2}\right), 31.8\left(\mathrm{CH}_{2}\right), 32.2\left(\mathrm{CH}_{2}\right), 34.2\left(\mathrm{CH}_{2}\right), 34.9(\mathrm{CH}), 35.0$ $(\mathrm{CH}), 35.8(\mathrm{C}), 36.8\left(\mathrm{CH}_{2}\right), 38.1\left(\mathrm{CH}_{2}\right), 39.9\left(\mathrm{CH}_{2}\right), 41.1$ (C), $44.7(\mathrm{CH}), 54.3(\mathrm{CH}), 55.5\left(\mathrm{CH}_{3}\right), 56.0(\mathrm{CH}), 61.7$ $(\mathrm{CH}), 68.3\left(\mathrm{CH}_{2}\right), 72.2(\mathrm{CH}), 79.7(\mathrm{CH}, \mathrm{C}-3), 82.0(\mathrm{C}), 82.3$ $(\mathrm{CH}), 116.8(\mathrm{C}) ; \mathrm{MS} \mathrm{m} / \mathrm{z}$ (rel intensity) $431\left(\mathrm{M}^{\mathrm{C}} \mathrm{KCH}_{2} \mathrm{OH}\right.$, 26), 413 (11), 287 (100); HRMS calcd for $\mathrm{C}_{27} \mathrm{H}_{43} \mathrm{O}_{4}$ 431.3161; found 431.3106. Anal. Calcd for $\mathrm{C}_{28} 8_{46} \mathrm{G}$, 72.69; H, 10.02. Found: C, 72.75; H, 9.92.

4.1.11. (22S, $23 S, 25 S)$-3b-Methoxy-5a-spirostan23,25- diol (29). A solution of compound 28 (47 mg, $0.1 \mathrm{mmol})$

in $\mathrm{CHCl}_{3}(2.4 \mathrm{~mL})$ was treated with $p$-TsOH $(5 \mathrm{mg}$ dissolved in $0.5 \mathrm{~mL} \mathrm{CHCl}_{3}$ ) and stirred at room temperature for $1 \mathrm{~h}$. The reaction mixture was neutralized with Amberjet $4400 \mathrm{OH}$, filtered and concentrated. Chromatotron chromatography (toluene-EtOAc, 60:40) of the residue afforded starting material 28 (30 mg, $0.06 \mathrm{mmol}, 60 \%)$ and com-

pound 29 (15 mg, $0.03 \mathrm{mmol}, 3 \mathrm{Q} \%)$. Compound 29: $\mathrm{mp}$ 214-216 8C (from acetone); [a] K92.2 (c 0.09); IR $3579 \mathrm{~cm}^{\mathrm{K} 1} ;{ }^{1} \mathrm{H}$ NMR $0.64(1 \mathrm{H}, \mathrm{m}), 0.80(6 \mathrm{H}, \mathrm{s}), 1.00(3 \mathrm{H}$, d, JZ7.0 Hz), $1.13(3 \mathrm{H}, \mathrm{s}), 1.56(1 \mathrm{H}, \mathrm{dd}, J Z 12.3$, $12.3 \mathrm{~Hz}), 2.05(1 \mathrm{H}$, ddd, JZ2.7, 5.3, $12.7 \mathrm{~Hz}), 2.56(1 \mathrm{H}$, dddd, $J Z 6.9,6.9,6.9,6.9 \mathrm{~Hz}), 3.11(1 \mathrm{H}$, dddd, $J Z 4.7,4.7$, $10.9,10.9 \mathrm{~Hz}), 3.23(1 \mathrm{H}, \mathrm{dd}, J Z 2.7,11.6 \mathrm{~Hz}), 3.33(3 \mathrm{H}, \mathrm{s})$, $3.62(1 \mathrm{H}, \mathrm{d}, J Z 11.5 \mathrm{~Hz}), 3.75(1 \mathrm{H}, \mathrm{dd}, J Z 5.3,11.6 \mathrm{~Hz})$, $4.45(1 \mathrm{H}$, ddd, $J Z 7.1,7.1,7.1 \mathrm{~Hz})$; NOE correlation from $23-\mathrm{H}$ to $20-\mathrm{H}$, to $21-\mathrm{H}_{3}$ and from $26-\mathrm{H}_{\mathrm{b}}$ to $24-\mathrm{H}_{\mathrm{a}} ;{ }^{13} \mathrm{C} \mathrm{NMR}$ $(100.6 \mathrm{MHz}) 12.3\left(\mathrm{CH}_{3}\right), 14.0\left(\mathrm{CH}_{3}\right), 16.6\left(\mathrm{CH}_{3}\right), 21.0$ $\left(\mathrm{CH}_{2}\right), 24.8\left(\mathrm{CH}_{3}\right), 27.8\left(\mathrm{CH}_{2}\right), 28.7\left(\mathrm{CH}_{2}\right), 31.7\left(\mathrm{CH}_{2}\right), 32.3$
$\left(\mathrm{CH}_{2}\right), 34.3\left(\mathrm{CH}_{2}\right), 34.9(\mathrm{CH}), 35.7(\mathrm{CH}), 35.9(\mathrm{C}), 36.9$ $\left(\mathrm{CH}_{2}\right), 40.1\left(\mathrm{CH}_{2}\right), 41.1(\mathrm{C}), 42.6\left(\mathrm{CH}_{2}\right), 44.8(\mathrm{CH}), 54.4$ $(\mathrm{CH}), 55.5\left(\mathrm{CH}_{3}\right), 56.3(\mathrm{CH}), 61.5(\mathrm{CH}), 64.3(\mathrm{CH}), 68.4$ $\left(\mathrm{CH}_{2}\right), 70.3(\mathrm{C}), 79.8(\mathrm{CH}), 82.1(\mathrm{CH}), 110.5(\mathrm{C}) ; \mathrm{MS} \mathrm{m} / \mathrm{z}$ (rel intensity) $462\left(\mathrm{M}^{\mathrm{C}}, 6\right), 444$ (3), 426 (3), 287 (100); HRMS calcd for $\mathrm{C}_{28} \mathrm{H}_{46} 62.3345$; found 462.3324 .

Anal. Calcd for $\mathrm{C}_{28} \mathrm{H}_{46} \mathrm{O}_{5}: \mathrm{C}, 72.69 ; \mathrm{H}, 10.02$. Found: $\mathrm{C}$, 72.67; H, 10.17.

4.1.12. (22S, $23 S, 25 R)$-3b-Methoxy-23-tertbutyldimethylsilyloxy-26-acetoxy-22,25-epoxy-5a-

fungstan (30)

butyldimethyl5a-furostan (31). A solu- tion of the alcohol 14-S (49 $\mathrm{mg}, 0.08 \mathrm{mmol})$ in cyclohexane $(8.6 \mathrm{~mL})$ containing (diacetoxyiodo)benzene $(52 \mathrm{mg}$,

$0.16 \mathrm{mmol})$ and iodine $(21 \mathrm{mg}, 0.08 \mathrm{mmol})$ was irradiated with a $80 \mathrm{~W}$ tungsten-filament lamp at $708 \mathrm{C}$ for $1 \mathrm{~h}$. The reaction mixture was then poured into aqueous solution of sodium thiosulfate $(10 \%)$ and extracted with $\mathrm{CH}_{2} \mathrm{Cl}_{2}$. The combined organic extracts were washed with brine, dried $\left(\mathrm{Na}_{2} \mathrm{SO}_{4}\right)$ and concentrated. Chromatotron chromatography (hexanes-EtOAc, 93:7) of the residue afforded compound 30 (14 mg, $0.02 \mathrm{mmol}, 28 \%$ ) and compound 31 (23 mg, $0.04 \mathrm{mmol}, 47 \%)$. Compound 30: $\mathrm{mp} 144.0-144.78 \mathrm{C}$ (from EtOAc); [a] $\mathrm{D}$ C 24 (c 0.10$)$; IR $1745 \mathrm{~cm}^{\mathrm{K} 1} ;{ }^{1} \mathrm{H}$ NMR 0.07 (3H, s), $0.08(3 \mathrm{H}, \mathrm{s}), 0.62(1 \mathrm{H}, \mathrm{m}), 0.80(3 \mathrm{H}, \mathrm{s})$, $0.89(3 \mathrm{H}, \mathrm{s}), 0.91(9 \mathrm{H}, \mathrm{s}), 1.13(3 \mathrm{H}, \mathrm{d}, J \mathrm{Z} 7.3 \mathrm{~Hz}), 1.34$ $(3 \mathrm{H}, \mathrm{s}), 1.67(1 \mathrm{H}, \mathrm{dd}, J Z 2.3,13.1 \mathrm{~Hz}), 2.06(3 \mathrm{H}, \mathrm{s}), 2.12$ (1H, dd, JZ5.12, $13.0 \mathrm{~Hz}), 2.33(1 \mathrm{H}, \mathrm{m}), 3.10$ (1H, dddd, $J Z 4.6,4.6,10.9,10.9 \mathrm{~Hz}), 3.33(3 \mathrm{H}, \mathrm{s}), 3.98(1 \mathrm{H}, \mathrm{d}$, $J Z 10.7 \mathrm{~Hz}), 4.03(1 \mathrm{H}, \mathrm{d}, J Z 10.7 \mathrm{~Hz}), 4.18(1 \mathrm{H}, \mathrm{dd}$, $J Z 2.3,5.0 \mathrm{~Hz}), 4.37(1 \mathrm{H}$, ddd, $J Z 7.4,7.4,7.4 \mathrm{~Hz})$; NOE correlation from $23-\mathrm{H}$ to $21-\mathrm{Me}$ and to $26-\mathrm{H}_{2} ;{ }^{13} \mathrm{C} \mathrm{NMR}$ (100.6 MHz) K5.2 $\left(\mathrm{CH}_{3}\right), \mathrm{K} 3.7\left(\mathrm{CH}_{3}\right), 12.3\left(\mathrm{CH}_{3}\right), 16.1$ $\left(\mathrm{CH}_{3}\right), 17.7\left(\mathrm{CH}_{3}\right), 17.8(\mathrm{C}), 20.9\left(\mathrm{CH}_{3}\right), 21.1\left(\mathrm{CH}_{2}\right), 25.2$ $\left(\mathrm{CH}_{3}\right), 25.9\left(3 ! \mathrm{CH}_{3}\right), 27.9 \quad\left(\mathrm{CH}_{2}\right), 28.8\left(\mathrm{CH}_{2}\right), 32.5$ $\left(\mathrm{CH}_{2}\right), 33.1\left(\mathrm{CH}_{2}\right), 34.3\left(\mathrm{CH}_{2}\right), 34.5(\mathrm{CH}), 35.1(\mathrm{C}), 36.9$ $\left(\mathrm{CH}_{2}\right), 39.2(\mathrm{CH}), 40.9\left(\mathrm{CH}_{2}\right), 41.2(\mathrm{C}), 42.7\left(\mathrm{CH}_{2}\right), 44.8$ $(\mathrm{CH}), 54.6(\mathrm{CH}), 54.9(\mathrm{CH}), 55.5\left(\mathrm{CH}_{3}\right), 62.8(\mathrm{CH}), 71.4$ $\left(\mathrm{CH}_{2}\right), 76.9(\mathrm{CH}), 79.8(\mathrm{CH}), 80.5(\mathrm{C}), 81.9(\mathrm{CH}), 121.1$ (C), $171.0(\mathrm{C}) ; \mathrm{MS} \mathrm{m} / \mathrm{z}$ (rel intensity) $618\left(\mathrm{M}^{\mathrm{C}}\right.$,

! 1), 561 (5), 198 (100); HRMS calcd for $\mathrm{C}_{36} \mathrm{H}_{62} \mathrm{O}_{6} \mathrm{Si}$ 618.4316; found 618.4321. Anal. Calcd for $\mathrm{C}_{36} \mathrm{H}_{62} \mathrm{O}_{6} \mathrm{Si}: \mathrm{C}$, 69.86; H, 10.10. Found: C, 69.80; H, 10.07. Compound 31: mp 157.0-157.8 8C (from EtOAc); [a] $]_{\mathrm{D}} \mathrm{K} 37.5$ (c 0.12); IR $1746 \mathrm{~cm}^{\mathrm{K} 1} ;{ }^{1} \mathrm{H}$ NMR $0.04(6 \mathrm{H}, \mathrm{s}), 0.60(1 \mathrm{H}, \mathrm{m}), 0.76$ $(3 \mathrm{H}$,

s), $0.77(3 \mathrm{H}, \mathrm{s}), 0.85(3 \mathrm{H}, \mathrm{d}, J Z 5.3 \mathrm{~Hz}), 0.87(9 \mathrm{H}, \mathrm{s}), 1.28$ $(3 \mathrm{H}, \mathrm{s}), 1.91(1 \mathrm{H}, \mathrm{dd}, J \mathrm{Z} 10.6,11.9 \mathrm{~Hz}), 2.02(3 \mathrm{H}, \mathrm{s}), 2.11$ (1H, m), 3.07 (1H, dddd, JZ4.5, 4.5, 10.8, $10.8 \mathrm{~Hz}), 3.29$ $(3 \mathrm{H}, \mathrm{s}), 3.77(1 \mathrm{H}, \mathrm{d}, J Z 11.2 \mathrm{~Hz}), 3.93(1 \mathrm{H}, \mathrm{d}, J Z 11.2 \mathrm{~Hz})$, 4.04 (1H, dd, JZ8.1, 10.4 Hz), 4.48 (1H, ddd, JZ7.3, 7.3, $7.3 \mathrm{~Hz}$ ); NOE correlation from $23-\mathrm{H}$ to $21-\mathrm{Me}$ and to $20-\mathrm{H}$; ${ }^{13} \mathrm{C}$ NMR (100.6 MHz) K4.9 $\left(\mathrm{CH}_{3}\right), \mathrm{K} 4.8\left(\mathrm{CH}_{3}\right), 12.2$ $\left(\mathrm{CH}_{3}\right), 13.9\left(\mathrm{CH}_{3}\right), 16.6\left(\mathrm{CH}_{3}\right), 18.2(\mathrm{C}), 20.8\left(\mathrm{CH}_{3}\right), 20.9$ $\left(\mathrm{CH}_{2}\right), 25.8\left(3 ! \mathrm{CH}_{3}\right), 26.0\left(\mathrm{CH}_{3}\right), 27.8\left(\mathrm{CH}_{2}\right), 28.7\left(\mathrm{CH}_{2}\right)$, $31.8\left(\mathrm{CH}_{2}\right), 32.2\left(\mathrm{CH}_{2}\right), 34.2\left(\mathrm{CH}_{2}\right), 35.0(2 ! \mathrm{CH}), 35.8$ (C), $36.9\left(\mathrm{CH}_{2}\right), 40.1\left(\mathrm{CH}_{2}\right), 40.4\left(\mathrm{CH}_{2}\right), 40.9(\mathrm{C}), 44.7$ $(\mathrm{CH}), 54.3(\mathrm{CH}), 55.4\left(\mathrm{CH}_{3}\right), 56.2(\mathrm{CH}), 61.6(\mathrm{CH}), 70.3$ $\left(\mathrm{CH}_{2}\right), 71.2(\mathrm{CH}), 78.3(\mathrm{C}), 79.7(\mathrm{CH}), 80.8(\mathrm{CH}), 116.5$ (C), 170.5 (C); MS m/z (rel intensity) $618\left(\mathrm{M}^{\mathrm{C}}, ! 1\right), 561$ (6), 545 (1), 75 (100); HRMS calcd for $\mathrm{C}_{36} \mathrm{H}_{62} \mathrm{O}_{6} \mathrm{Si}$ 618.4316; found 618.4303. Anal. Calcd for $\mathrm{C}_{36} \mathrm{H}_{62} \mathrm{O}_{6} \mathrm{Si}: \mathrm{C}$, 69.86; H, 10.10. Found: C, 69.95; H, 10.13. 
4.1.13. (22S,23S,25R)-3b-Methoxy-22,25-epoxy5a-furo- stan-23,26-diol (32). To a solution of compound $30(55 \mathrm{mg}$,

$0.09 \mathrm{mmol})$ in THF $(12.6 \mathrm{~mL})$ was added $\mathrm{TBAF}(0.42 \mathrm{~mL}$, $0.42 \mathrm{mmol}, 1.0 \mathrm{M}$ in THF) and stirred at room temperature for $3 \mathrm{~h}$. The mixture was then poured into aqueous saturated solution of $\mathrm{NaHCO}_{3}$ and extracted with $\mathrm{CH}_{2} \mathrm{Cl}_{2}$. The organic extracts were washed with brine, dried $\left(\mathrm{Na}_{2} \mathrm{SO}_{4}\right)$ and concentrated. A solution of the residue in $\mathrm{MeOH}$ (26 $\mathrm{mL})$ containing $\mathrm{KOH}(0.9 \mathrm{~g})$ was stirred at room temperature for $1.5 \mathrm{~h}$. The mixture was poured into water and extracted with EtOAc. The combined extracts were washed with brine, dried $\left(\mathrm{Na}_{2} \mathrm{SO}_{4}\right)$ and concentrated. Chromatotron chromatography (hexanes-EtOAc, 7:3) afforded compound 32 (41 mg, $0.09 \mathrm{mmol}, 99 \%)$ : $\mathrm{mp}$ 145.1-146.7 8C (from AcOEt); [a] $]_{\mathrm{D}} \mathrm{C} 7.8$ (c 0.09); IR $3629,3448 \mathrm{~cm}^{\mathrm{K} 1} ;{ }^{1} \mathrm{H}$ NMR $0.62(1 \mathrm{H}, \mathrm{m}), 0.79(3 \mathrm{H}, \mathrm{s}), 0.93$ $(3 \mathrm{H}, \mathrm{s}), 1.25(3 \mathrm{H}, \mathrm{d}, J Z 7.3 \mathrm{~Hz}), 1.31(3 \mathrm{H}, \mathrm{s}), 1.55(1 \mathrm{H}, \mathrm{dd}$, $J Z 0.0,13.5 \mathrm{~Hz}), 2.46(1 \mathrm{H}$, dddd, $J Z 3.1,7.3,7.3,7.3 \mathrm{~Hz})$, $2.56(1 \mathrm{H}, \mathrm{dd}, J Z 4.6,13.6 \mathrm{~Hz}), 3.11(1 \mathrm{H}, \mathrm{dddd}, J Z 4.6,4.6$, 11.0, $11.0 \mathrm{~Hz}), 3.31(1 \mathrm{H}, \mathrm{d}, J Z 10.3 \mathrm{~Hz}), 3.32(3 \mathrm{H}, \mathrm{s}), 3.54$ $(1 \mathrm{H}, \mathrm{d}, J Z 11.3 \mathrm{~Hz}), 4.15(1 \mathrm{H}, \mathrm{dd}, J Z 0.0,4.5 \mathrm{~Hz}), 4.53$ (1H, ddd, $J Z 7.1,7.1,9.3 \mathrm{~Hz}$ ); NOE correlation from $23-\mathrm{H}$ to $21-\mathrm{H}_{3} ;{ }_{2}^{13} \mathrm{C}$ NMR $12.2\left(\mathrm{CH}_{3}\right),{ }_{2} 15.9\left(\mathrm{CH}_{3}\right)_{2} 18.7\left(\mathrm{CH}_{3}\right)$, $20.9(\mathrm{CH}), 25.8(\mathrm{CH}), 27.8(\mathrm{CH}), 28.6(\mathrm{CH}), 32.3(\mathrm{CH})$, $33.5\left(\mathrm{CH}_{2}\right), 34.2\left(\mathrm{CH}_{2}\right), 34.4(\mathrm{CH}), 35.8(\mathrm{C}), 36.8\left(\mathrm{CH}_{2}\right)$, $38.4(\mathrm{CH}), 39.1\left(\mathrm{CH}_{2}\right), 40.5\left(\mathrm{CH}_{2}\right), 41.4(\mathrm{C}), 44.7(\mathrm{CH})$, $54.5(\mathrm{CH}), 55.0(\mathrm{CH}), 55.4\left(\mathrm{CH}_{3}\right), 64.6(\mathrm{CH}), 68.3\left(\mathrm{CH}_{2}\right)$, $76.7(\mathrm{CH}), 79.8(\mathrm{CH}), 83.1(\mathrm{CH}), 84.4(\mathrm{C}), 120.6(\mathrm{C})$; MS $\mathrm{m} / \mathrm{z}$ (rel intensity) $444\left(\mathrm{M}^{\mathrm{C}}-\mathrm{H}_{2} \mathrm{O}, 5\right), 426$ (34), 287 (100); HRMS calcd for $\mathrm{C}_{28} \mathrm{H}_{44} \mathrm{O}_{4} 444.3240$; found 444.3239. Anal. Calcd for $\mathrm{C}_{28} \mathrm{H}_{46} \mathrm{O}_{5}: \mathrm{C}, 72.69 ; \mathrm{H}, 10.02$. Found: C, $72.85 ; \mathrm{H}, 9.74$.

4.1.14. (22R,23S,25R)-3b-Methoxy-22,25-epoxy5a-furo- stan-23,26-diol (33). To a solution of compound 31 (108 $\mathrm{mg}, 0.17 \mathrm{mmol})$ in THF $(25 \mathrm{~mL})$ was added TBAF (0.84 mL, $0.84 \mathrm{mmol}, 1.0 \mathrm{M}$ in THF) and stirred at room temperature for $1 \mathrm{~h}$. The mixture was then poured into aqueous saturated solution of $\mathrm{NaHCO}_{3}$ and extracted with $\mathrm{CH}_{2} \mathrm{Cl}_{2}$. The organic extracts were washed with brine, dried $\left(\mathrm{Na}_{2} \mathrm{SO}_{4}\right)$ and concentrated. A solution of the residue in $\mathrm{MeOH}(50 \mathrm{~mL})$ containing $\mathrm{KOH}(1.7 \mathrm{~g})$ was stirred at room temperature for $5 \mathrm{~h}$. The mixture was poured into water and extracted with EtOAc. The combined extracts were washed with brine, dried $\left(\mathrm{Na}_{2} \mathrm{SO}_{4}\right)$ and concentrated. Chromatotron chromatography (hexanes-EtOAc, 7:3) afforded compound 33 (74 mg, 0.16 mmol, 91\%): $\mathrm{mp}$ 198.5-199.7 8C (from $n$ - hexaneEtOAc); [a] $]_{\mathrm{D}} \mathrm{K} 47.7$ (c 0.13); IR $3567 \mathrm{mmolcm}^{\mathrm{K} 1}$; ${ }^{1} \mathrm{H}$ NMR $0.64(1 \mathrm{H}, \mathrm{m}), 0.77(3 \mathrm{H}, \mathrm{s}), 0.79(3 \mathrm{H}, \mathrm{s}), 0.95(3 \mathrm{H}, \mathrm{d}$, $J Z 7.0 \mathrm{~Hz}), 1.26(3 \mathrm{H}, \mathrm{s}), 1.69(1 \mathrm{H}, \mathrm{dd}, J Z 9.8,12.4 \mathrm{~Hz})$, $2.32(1 \mathrm{H}, \mathrm{dd}, J Z 8.5,12.4 \mathrm{~Hz}), 3.10$ (1H, dddd, $J Z 4.6,4.6$, 11.0, $11.0 \mathrm{~Hz}), 3.30(1 \mathrm{H}, \mathrm{d}, J Z 11.0 \mathrm{~Hz}), 3.32(3 \mathrm{H}, \mathrm{s}), 3.39$ $(1 \mathrm{H}, \mathrm{d}, J Z 11.3 \mathrm{~Hz}), 4.03(1 \mathrm{H}, \mathrm{ddd}, J Z 9.0,9.0,9.0 \mathrm{~Hz})$, $4.55(1 \mathrm{H}$, ddd, $J Z 6.6,6.6,8.7 \mathrm{~Hz})$; NOE correlation from 23 to $21-\mathrm{Me}$, to $20-\mathrm{H}$ and to $26-\mathrm{H}_{2} ;{ }^{13} \mathrm{C}$ NMR $(100.6 \mathrm{MHz})$ 12.2 $\left(\mathrm{CH}_{3}\right), 14.3\left(\mathrm{CH}_{3}\right), 16.4\left(\mathrm{CH}_{3}\right), 20.9\left(\mathrm{CH}_{2}\right), 25.6\left(\mathrm{CH}_{3}\right)$, $27.8\left(\mathrm{CH}_{2}\right), 28.7\left(\mathrm{CH}_{2}\right), 31.8\left(\mathrm{CH}_{2}\right), 32.2\left(\mathrm{CH}_{2}\right), 34.3\left(\mathrm{CH}_{2}\right)$, $34.9(\mathrm{CH}), 35.5(\mathrm{CH}), 35.8(\mathrm{C}), 36.8\left(\mathrm{CH}_{2}\right), 40.0\left(\mathrm{CH}_{2}\right)$, $41.0(\mathrm{C}), 41.3\left(\mathrm{CH}_{2}\right), 44.7(\mathrm{CH}), 54.3(\mathrm{CH}), 55.5\left(\mathrm{CH}_{3}\right)$, $56.1(\mathrm{CH}), 61.8(\mathrm{CH}), 69.8\left(\mathrm{CH}_{2}\right), 72.2(\mathrm{CH}), 79.7(\mathrm{CH})$, $81.3(\mathrm{CH}), 81.3(\mathrm{C}), 116.7(\mathrm{C})$; MS $\mathrm{m} / \mathrm{z}$ (rel intensity) 444 $\left(\mathrm{M}^{\mathrm{C}}-\mathrm{H}_{2} \mathrm{O}, 5\right), 426$ (27), 287 (100); HRMS calcd for
$\mathrm{C}_{28} \mathrm{H}_{44} \mathrm{O}_{4}$ 444.3240; found 444.3252. Anal. Calcd for $\mathrm{C}_{28} \mathrm{H}_{46} \mathrm{O}_{5}$ : C, 72.69; H, 10.02. Found: C, 72.72; H, 9.98.

4.1 .15

$(22 S, 23 S, 25 R)-3 \mathrm{~b}-$ Methoxy-22,26epoxy-5a-

spirostan-23,25-diol (34). A solution of compound 33 (46 mg, $0.1 \mathrm{mmol})$ in $\mathrm{CHCl}_{3}(2.5 \mathrm{~mL})$ was treated with $p$ TsOH (5 mg dissolved in $0.5 \mathrm{~mL} \mathrm{CHCl}_{3}$ ) and stirred at room temperature for $24 \mathrm{~h}$. The reaction mixture was neutralized with Amberjet $4400 \mathrm{OH}$, filtered and concentrated. Chromatotron chromatography (toluene-EtOAc, 65:35) of the residue afforded starting material $33(31 \mathrm{mg}, 0.06 \mathrm{mmol}$, $60 \%$ ) and compound 34 (13 $\mathrm{mg}, 0.03 \mathrm{mmol}, 30 \%)$. Compound 34: mp 263-263.5 8C (from acetone); [a $]_{\mathrm{D}}$ $\mathrm{K} 132.8$ (c 0.07); IR $3576 \mathrm{~cm}^{\mathrm{K} 1}$; ${ }^{1} \mathrm{H}$ NMR $0.64(1 \mathrm{H}, \mathrm{m})$, $0.79(3 \mathrm{H}, \mathrm{s}), 0.80(3 \mathrm{H}, \mathrm{s}), 0.95(3 \mathrm{H}, \mathrm{d}, J Z 7.0 \mathrm{~Hz}), 1.30(3 \mathrm{H}$, s), 1.99 (1H, ddd, $J Z 5.5,7.6,12.3 \mathrm{~Hz}), 2.07$ (1H, ddd, $J Z$ $2.5,5.2,11.6 \mathrm{~Hz}), 2.50(1 \mathrm{H}$, dddd, $J Z 7.0,7.0,7.0,7.0 \mathrm{~Hz})$, $3.11(1 \mathrm{H}$, dddd, $J Z 4.6,4.6,10.9,10.9 \mathrm{~Hz}), 3.19(1 \mathrm{H}$, dd, $J Z 2.5,10.4 \mathrm{~Hz}), 3.33(3 \mathrm{H}, \mathrm{s}), 3.49(1 \mathrm{H}, \mathrm{dd}, J Z 5.3$, $11.7 \mathrm{~Hz}), 3.50(1 \mathrm{H}, \mathrm{d}, J Z 10.2 \mathrm{~Hz}), 4.46(1 \mathrm{H}, \mathrm{ddd}, J Z 7.1$, $7.1,8.7 \mathrm{~Hz}$ ); NOE correlation from $23-\mathrm{H}$ to $20-\mathrm{H}$, to $21-\mathrm{H}_{3}$; ${ }^{13} \mathrm{C}$ NMR $(100.6 \mathrm{MHz}) 12.3\left(\mathrm{CH}_{3}\right), 13.9\left(\mathrm{CH}_{3}\right), 16.6\left(\mathrm{CH}_{3}\right)$, $21.0\left(\mathrm{CH}_{2}\right), 25.0\left(\mathrm{CH}_{3}\right), 27.8\left(\mathrm{CH}_{2}\right), 28.7\left(\mathrm{CH}_{2}, 31.6\left(\mathrm{CH}_{2}\right)\right.$, $32.3\left(\mathrm{CH}_{2}\right), 34.3\left(\mathrm{CH}_{2}\right), 34.9(\mathrm{CH}), 35.6(\mathrm{CH}), 35.9(\mathrm{C})$, $36.9\left(\mathrm{CH}_{2}\right), 40.1\left(\mathrm{CH}_{2}\right), 41.0(\mathrm{C}), 44.6\left(\mathrm{CH}_{2}\right), 44.8(\mathrm{CH})$, $54.4(\mathrm{CH}), 55.5\left(\mathrm{CH}_{3}\right), 56.2(\mathrm{CH}), 61.5(\mathrm{CH}), 65.8(\mathrm{CH})$, $68.2\left(\mathrm{CH}_{2}\right), 68.9(\mathrm{C}), 79.8(\mathrm{CH}), 82.0(\mathrm{CH}), 110.0(\mathrm{C})$; MS $\mathrm{m} / \mathrm{z}$ (rel intensity) 462 ( $\mathrm{M}^{\mathrm{C}}, 1$ ), 444 (2), 426 (5), 287 (100); HRMS calcd for C $\mathrm{H} Q 463.3345$; found 462.3336 .

Anal. Calcd for $\mathrm{C}_{28} \mathrm{H}_{46} \mathrm{O}_{5}: \mathrm{C}, 72.69 ; \mathrm{H}, 10.02$. Found: $\mathrm{C}$, 72.89; H, 10.13 .

4.1.16. (22S, $23 S, 25 R)$-3b-Methoxy-23-acetoxy-5aspiro- stan-23,25-diol (35). The compound 34 (4 mg, $8.6 ! 10^{\mathrm{K} 3} \mathrm{mmol}$ ) was acetylated with $\mathrm{Ac}_{2} \mathrm{O}$ and pyridine to give after chromatography (hexanes-EtOAc, $85: 15)$ compound $35\left(3 \mathrm{mg}, 5.9 ! 10^{\mathrm{K} 3} \mathrm{mmol}, 68 \%\right)$ : amorphous; [a] $]_{\mathrm{D}} \mathrm{K} 72.0$ (c 0.05); IR 3434, $1745 \mathrm{~cm}^{\mathrm{K} 1} ;{ }^{1} \mathrm{H}$ NMR $0.64(1 \mathrm{H}, \mathrm{m}), 0.78(3 \mathrm{H}, \mathrm{s}), 0.80(3 \mathrm{H}, \mathrm{s}), 0.96(3 \mathrm{H}, \mathrm{d}$, $J Z 7.0 \mathrm{~Hz}), 1.37(3 \mathrm{H}, \mathrm{s}), 2.04(3 \mathrm{H}, \mathrm{s}), 2.07(1 \mathrm{H}, \mathrm{m}), 3.12$ $(1 \mathrm{H}$, dddd, $J Z 4.6,4.6,10.9,10.9 \mathrm{~Hz}), 3.22(1 \mathrm{H}, \mathrm{dd}$, $J Z 2.0,10.5 \mathrm{~Hz}), 3.33(3 \mathrm{H}, \mathrm{s}), 3.60(1 \mathrm{H}, \mathrm{d}, J Z 10.6 \mathrm{~Hz})$, $4.45(1 \mathrm{H}$, ddd, $J Z 7.7,7.7,7.7 \mathrm{~Hz})$,

$4.84(1 \mathrm{H}, \mathrm{dd}, J Z 5.3,11.9 \mathrm{~Hz})$; NOE correlation from $23-\mathrm{H}$ to $20-\mathrm{H}$, to $21-\mathrm{H}_{3}$ and to $27-\mathrm{H}_{3} ;{ }^{13} \mathrm{C}$ NMR $(100.6 \mathrm{MHz}) 12.3$ $\left(\mathrm{CH}_{3}\right), 13.9\left(\mathrm{CH}_{3}\right), 16.1\left(\mathrm{CH}_{3}\right), 20.9\left(\mathrm{CH}_{2}\right), 21.0\left(\mathrm{CH}_{3}\right), 25.3$ $\left(\mathrm{CH}_{3}\right), 27.9\left(\mathrm{CH}_{2}\right), 28.7\left(\mathrm{CH}_{2}\right), 31.6\left(\mathrm{CH}_{2}\right), 32.3\left(\mathrm{CH}_{2}\right), 34.3$ $\left(\mathrm{CH}_{2}\right), 35.2\left(\mathrm{CH}_{2}, 35.9(\mathrm{C}), 36.0(\mathrm{CH}), 36.9\left(\mathrm{CH}_{2}\right), 39.9\right.$ $(\mathrm{CH}), 40.3(\mathrm{CH}), 41.1(\mathrm{C}), 44.8(\mathrm{CH}), 54.4(\mathrm{CH}), 55.5$ $\left(\mathrm{CH}_{3}\right), 56.3(\mathrm{CH}), 61.5(\mathrm{CH}), 66.7(\mathrm{CH}), 68.1\left(\mathrm{CH}_{2}\right), 69.0$ (C), $79.8(\mathrm{CH}), 81.6(\mathrm{CH}), 108.2(\mathrm{C}), 170.4(\mathrm{C}) ; \mathrm{MS} \mathrm{m} / \mathrm{z}$ (rel intensity) $504\left(\mathrm{M}^{\mathrm{C}}, 3\right), 486$ (6), 444 (31), 287 (100); HRMS calcd for $\mathrm{C}_{30} \mathrm{H}_{48} \mathrm{O}_{6}$ 504.3451; found 504.3418. Anal. Calcd for $\mathrm{C}_{30} \mathrm{H}_{48} \mathrm{O}_{6}$ : C, 71.39; H, 9.59. Found: $\mathrm{C}, 71.27 ; \mathrm{H}, 9.84$.

\section{Acknowledgements}

This work was supported by the Investigation Programmes nos. BQU2000-0650 and BQU2001-1665 of the Direccio'n General de Investigacio'n Cient' ifica y Te'cnica, Spain. I.P.M. thanks the I3P-CSIC Program for a fellowship. 


\section{Supplementary data}

Detailed experimental procedures, and spectral and analytical data for compounds 6-R, 6-S, 7-R, 7-S, 8-R, 8-S, 9-R, 9- $S, 10-R, 10-S, 11-S, 12-R, 12-S, 13-S, 14-R$, and $14-S(15$

pages) are provided. A figure with the pseudorotational wheels for $\mathrm{E}$ and F-rings is also included.

Supplementary data associated with this article can be found, in the online version, at doi:10.1016/j.tet.2005.01. $\underline{077}$

\section{References and notes}

1. (a) Pettit, G. R.; Inoue, M.; Kamano, Y.; Herald, D. L.; Arm, C.; Dufresne, C.; Christie, N. D.; Schmidt, J. M.; Doubek, D. L.; Krupa, T. S. J. Am. Chem. Soc. 1988, 110, 20062007.

(b) Recent publication: Pettit, G. R.; Tan, R.; Xu, J.-P.; Ichihara, Y.; Williams, M. D.; Boyd, M. R. J. Nat. Prod. 1998, 61, 955-958 and references cited therein.

2. (a) Fukuzawa, S.; Matsunaga, S.; Fusetani, N. J. Org. Chem. 1994, 59, 6164-6166. (b) Fukuzawa, S.; Matsunaga, S.; Fusetani, N. J. Org. Chem. 1995, 60, 608-614. (c) Fukuzawa, S.; Matsunaga, S.; Fusetani, N. Tetrahedron 1995, 51, 67076716. (d) Fukuzawa, S.; Matsunaga, S.; Fusetani, N. J. Org. Chem. 1997, 62, 4484-4491.

3. (a) Pettit, G. R.; Xu, J.-P.; Williams, M. D.; Christie, N. D.; Doubek, D. L.; Schmidt, J. M.; Boyd, M. R. J. Nat. Prod. 1994,

57, 52-63. (b) LaCour, T. G.; Guo, C.; Ma, S.; Jeong, J. U.; Boyd, M. R.; Matsunaga, S.; Fusetani, N.; Fuchs, P. L. Bioorg. Med. Chem. Lett. 1999, 9, 2587-2592. (c) Dirsch, V. M.; Muller, I. M.; Eichhorst, S. T.; Pettit, G. R.; Kamano, Y.; Inoue, M.; Xu, J. P.; Ichihara, Y.; Wanner, G.; Vollmar, A. M. Cancer Res. 2003, 63, 8869-8876. (d) Komiya, T.; Fusetani,

N.; Matsunaga, S.; Kubo, A.; Kaye, F. J.; Kelley, M. J.; Tamura, K.; Yoshida, M.; Fukuoka, M.; Nakagawa, K. Cancer Chemother. Pharm. 2003, 51, 202-208.

4. (a) Heathcock, C. H.; Smith, S. C. J. Org. Chem. 1994, 59, 6828-6839. (b) Dro"gemu"ller, M.; Jautelat, R.; Winterfeldt, E. Angew. Chem., Int. Ed. Engl. 1996, 35, 1572-1574. (c) LaCour,

T. G.; Guo, C.; Bhandaru, S.; Boyd, M. R.; Fuchs, P. L. J. Am. Chem. Soc. 1998, 120, 692-707. (d) Dro"gemu"ller, M.; Flessner, T.; Jautelat, R.; Scholz, U.; Winterfeldt, E. Eur. J. Org. Chem. 1998, 2811-2831. (e) Jautelat, R.; Müller-Fahrnow, A.; Winterfeldt, E. Chem. Eur. J. 1999, 5, 12261233. (f) Kim, S.; Sutton, S. C.; Guo, C.; LaCour, T. G.; Fuchs, P. L. J. Am. Chem. Soc. 1999, 121, 2056-2070.

(g) Jeong, J. U.; Guo, C.; Fuchs, P. L. J. Am. Chem. Soc. 1999, 121, 2071-2084. (h) Li, W.; Fuchs, P. L. Org. Lett. 2003, 5, 2849-2852. (i) Jeong, J. U.; Sutton, S. C.; Kim, S.; Fuchs, P. L. J. Am. Chem. Soc. 1995, 117, 10157-10158. (j) For a short review see: Ganesan, A. Angew. Chem., Int. Ed. Engl. 1996, 35, 611-615.

5. (a) Li, W.; Fuchs, P. L. Org. Lett. 2003, 5, 2853-2856. (b) Lee,

J. S.; Fuchs, P. L. Org. Lett. 2003, 5, 3619-3622. (c) Lee, J. S.; Fuchs, P. L. Org. Lett. 2003, 5, 2247-2250. (d) Lee, S.;

Fuchs, P. L. J. Am. Chem. Soc. 2002, 124, 13978-13979. (e)

Flessner,

T.; Ludwing, V.; Siebeneicher, H.; Winterfeldt, E. Synthesis 2002, 1373-1378. (f) Li, W.; LaCour, T. G.; Fuchs, P.L.
J. Am. Chem. Soc. 2002, 124, 4548-4549. (g) Lee, S.; Fuchs, P. L. Org. Lett. 2002, 3, 317-318. (h) LaCour, T. G.; Tong, Z.; Fuchs, P. L. Org. Lett. 1999, 1, 1815-1818.

6. LaCour, T. G.; Guo, C.; Boyd, M. R.; Fuchs, P. L. Org. Lett. 2000, 2, 33-36.

7. Isolation (a) : Kubo, S.; Mimaki, Y.; Terao, M.; Sashida, Y.; Nikaido, T.; Ohmoto, T. Phytochemistry 1992, 31, 39693973. Synthesis (b): Guo, C.; Fuchs, P. L. Tetrahedron Lett. 1998, 39, 1099-1102. (c) Deng, S.; Yu, B.; Lou, Y.; Hui, Y. J. Org. Chem. 1999, 64, 202-208. (d) Morzycki, J. W.; Gryszkiewicz, A.; Jastrzebska, I. Tetrahedron Lett. 2000, 41, 3751-3754. (e) Yu, W.; Jin, Z. J. Am. Chem. Soc. 2001, 123, 3369-3370. (f) Yu, W.; Jin, Z. J. Am. Chem. Soc. 2002, 124, 6576-6583.

8. Guo, C.; LaCour, T. G.; Fuchs, P. L. Bioorg. Med. Chem. Lett. 1999, 9, 419-424.

9. Part of this work was previously published as a preliminary communication: Betancor, C.; Freire, R.; Pe'rez-Mart'in, I.; Prange', T.; Sua'rez, E. Org. Lett. 2002, 4, 1295-1297.

10. (a) Majetich, G.; Wheless, K. Tetrahedron 1995, 51, 70957129. (b) Feray, L.; Kuznetsov, N.; Renaud, P. In Hydrogen Atom Abstraction; Renaud, P., Sibi, M. P., Eds.; Radicals in Organic Synthesis; Wiley-VCH: Weinheim, 2001; Vol. 2, pp 246-278. (c) Robertson, J.; Pillai, J.; Lush, R. K. Chem. Soc. Rev. 2001, 30, 94-103. (d) Togo, H.; Katohgi, M. Synlett 2001, 565-581.

11. (a) Mart'in, A.; Salazar, J. A.; Sua'rez, E. J. Org. Chem. 1996, 61, 3999-4006. (b) Dorta, R. L.; Mart'in, A.; Salazar, J. A.; Sua'rez, E.; Prange', T. J. Org. Chem. 1998, 63, 2251-2261.

12. See for example: pairs of ritterazines $A / D, B / F($ Ref. $\underline{2 b}$ ) and N/O, P/Q, R/S and W/X (Ref. $\underline{2 c}$ ).

13. (a) Barton, D. H. R.; Sammes, P. G.; Taylor, M. V.; Werstiuk, E. J. Chem. Soc. (C) 1970, 1977-1981. (b) Gonza'lez, A. G.; Freire, R.; Garc'ia-Estrada, M. G.; Salazar, J. A.; Sua'rez, E. Tetrahedron 1972, 28, 1289-1297. (c) Gonza'lez, A. G.;Freire, R.; Garc'1a-Estrada, M. G.; Salazar, J. A.; Sua'rez, E. Anal. Quim. 1971, 67, 903-905.

14. Oikawa, M.; Oikawa, H.; Ichihara, A. Tetrahedron 1995, 51, 6237-6254.

15. (a) Grieco, P. A.; Gilman, S.; Nishizawa, M. J. Org. Chem. 1976, 41, 1485-1486. (b) Grieco, P. A.; Takigawa, T.; Schillinger, W. J. J. Org. Chem. 1980, 45, 2247-2251.

16. For similar unsuccessful results on a related olefin using (DHQ) ${ }_{2}$ PHAL as ligand Sharpless, K. B.; Amberg, W.; Bennani, Y. L.; Crispino, G. A.; Hartung, J.; Jeong, K. S.; Kwong, H. L.; Morikawa, K.; Wang, Z. M.; Xu, D.; Zhang, X. L. J. Org. Chem. 1992, 57, 2768-2771 see: Refs. 5c,17b.

17. Corey, E. J.; Jardine, P. D.; Virgil, S.; Yuen, P.-W.; Connell, R. D. J. Am. Chem. Soc. 1989, 111, 9243-9244.

18. For studies of the influence of polar factors on intermolecular hydrogen abstraction reactions see: (a) Beckwith, A. L. J.; Zavitsas, A. A. J. Am. Chem. Soc. 1995, 117, 607-614.

(b) Zavitsas, A. A.; Chatgilialoglu, C. J. Am. Chem. Soc. 1995, 117, 10645-10654. (c) Kaushal, P.; Mok, P. L. H.; Roberts, B. P. J. Chem. Soc., Perkin Trans. 2 1990, 1663-1670.

19. Although we have no experimental support for this reasoning, it is strongly supported by previous results on related models. For examples of the influence of the polarity of the substituents on the 1,5-hydrogen atom tranfer reaction compared with competing b-fragmentation of alkoxyl radicals, see: (a) Allen, P. R.; Brimble, M. A.; Fare`s, F. A. J. Chem. Soc., Perkin Trans. 1 1998, 2403-2411. (b) Lee, S.; LaCour, T. G.; Lantrip, D.; Fuchs, P. L. Org. Lett. 2002, 4, 313-316.

20. For example, irradiation at $\mathrm{H}-23$ in each of the different 
diastereoisomers results in enhancement of the following protons: 17 (21-Me and 26- $\left.\mathrm{H}_{2}\right), 18$ (18-Me and 20-H), 22 (21- Me and 27-Me), 23 (18-Me, 20-H, and 27-Me), 27 (21Me), 28

(20H, 21-Me, and 27-Me), 32 (21-Me), 33 (21-Me and 26- $\mathrm{H}_{2}$ ).

21. Crystallographic data (excluding structure factors) for the structures 18, 23, 29, and 34 in this paper have been deposited with the Cambridge Crystallographic Data Centre as supple- mentary publication numbers CCDC-176076, CCDC-176077, CCDC-243668, CCDC-243669 respectively. Copies of the data can be obtained, free of charge, on application to CCDC, 12 Union Road, Cambridge CB2 1EZ, UK [fax: C44 (0) 1223- 336033 or e-mail: deposit@ccdc.cam.ac.uk].

22. Acid-catalyzed spiroketal equilibration in 23-deoxy compounds: (a) Thompson, M. J.; Scheer, I.; Mosettig, E. J. Am. Chem. Soc. 1959, 81, 5222-5224. (b) Thompson, M. J.; Scheer, I.;

Mosettig, E. J. Am. Chem. Soc. 1959, 81, 5225-5230. (c)

Gonza'lez, A. G.;

Freire, R.; Salazar, J. A.; Sua'rez, E. Phytochemistry 1971, 10, 1339-1346. (d) Gonza'lez, A. G.; Freire, R.; Salazar, J. Anal. Quim. 1972, 68, 1021-1027. (e) Jeong, J. U.; Fuchs, P. L.

Tetrahedron Lett. 1994, 35, 5385-5388.

23. No compounds of the 1,6-dioxaspiro[4.5]decane type belonging to the $(22 S)$-spirostan, $(20 R, 22 R)$-spirostan (cyclopseudoiso), or $(20 R, 22 S)$-spirostan (cyclopseudoneo) series of spirostan sapogenins, which are theoretically possible through an oxocarbenium ion via an intermediate of furost- 20(22)-en sapogenin, were detected during these acid- catalyzed experiments. (a) Fieser, L. F.; Fieser, M. In Steroids; Reinhold Publishing Corp: New York, 1959. (b) IUPAC-IUB Revised Tentative Rule for Nomenclature of Steroids Verkade, P. E.; Hoffmann-Ostenhof, O. J. Org. Chem. 1969, 34, 15171532.

24. CS Chem3D Ultra v. 6.0, CambridgeSoft Corp.: Cambridge, MA.

25. Dihedral angles consistent with these configurations are observed in the X-ray crystallographic structures of 17 (X-ray) $\left(\mathrm{H}_{23}-\mathrm{C}_{23}-\right.$
$\mathrm{C}_{24}-\mathrm{H}_{24 \mathrm{a}} \mathrm{Z} 154.4$ and $\left.\mathrm{H}_{23}-\mathrm{C}_{23}-\mathrm{C}_{24}-\mathrm{H}_{24 \mathrm{~b}} \mathrm{Z} 34.88\right)$ and 18

$\left(\mathrm{H}_{23}-\mathrm{C}_{23}-\mathrm{C}_{24}-\mathrm{H}_{24 \mathrm{a}} \mathrm{Z} 29.7\right.$ and $\left.\mathrm{H}_{23}-\mathrm{C}_{23}-\mathrm{C}_{24}-\mathrm{H}_{24 \mathrm{~b}} \mathrm{Z} 90.68\right)$.

26. (a) Fuchs, B. Top. Stereochem. 1978, 10, 1-94. (b) Romers, C.; Altona, C.; Buys, H. R.; Havinga, E. Top. Stereochem. 1969, 4, 36-97.

27. Data were taken from the X-ray analysis of $(22 S, 23 R, 25 S)$ 3b,12b-diacetoxy-22,25-epoxy-5a-furostan-23,26-diol.Jeong, J. U.; Fuchs, P. L. J. Am. Chem. Soc. 1994, 116, 773-774.

28. The five endocyclic dihedral angles $f_{j}(J Z 0-4)$ were obtained from the X-ray cif files using Mercury v. 1.2.1 (Cambridge Crystallographic Data Centre, 12 Union Road, Cambridge CB2 1EZ, UK) (http://www.ccdc.cam.ac.uk/mercury/).

29. (a) Houseknecht, J. B.; Altona, C.; Hadad, C. M.; Lowary, T. L. J. Org. Chem. 2002, 67, 4647-4651. (b) Altona, C.;

Sundaralingam, M. J. Am. Chem. Soc. 1973, 95, 2333-2344. (c) Altona, C.; Sundaralingam, M. J. Am. Chem. Soc. 1972, 94, 8205-8212.

30. IUPAC: Nomenclature of Carbohydrates, IUPAC, (http:// www.chem.qmul.ac.uk/iupac/2carb/). Rule 2-Carb-7.1.

31. Coupling constants were measured using the HLA equation as implemented in MacroModel v. 7.0: Haasnoot, C. A. G.; de Leeuw, F. A. A. M.; Altona, C. Tetrahedron 1980, 36, 2783 2792.

32. For an early conformational study of the F-ring of natural spirostan sapogenins deoxygenated at C-23 see: Callow, R. K.; James, V. H. T.; Kennard, O.; Page, J. E.; Paton, P. N.; Riva di Sanseverino, L. J. Chem. Soc. (C) 1966, 288-297.

33. Shortly before submitting our preliminary communication (Ref. 9) we became aware of the studies by Prof. P.L. Fuchs in the ASAP section of Organic Letters (Refs. 5g, 19b, see also Ref. 5c) where IHA reactions are used to prepare related dioxaspiro compounds.

34. (a) Westhof, E.; Sundaralingam, M. J. Am. Chem. Soc. 1983, 105, 970-976. (b) Levitt, M.; Warshel, A. J. Am. Chem. Soc. 1978, 100, 2607-2613. 